\title{
ANALYSIS OF ADAPTIVE RESPONSE TO DOSING PROTOCOLS FOR BIOFILM CONTROL*
}

\author{
BARBARA SZOMOLAY ${ }^{\dagger}$, ISAAC KLAPPER ${ }^{\ddagger}$, AND MARTIN DINDOS ${ }^{\S}$
}

\begin{abstract}
Biofilms are sessile populations of microbes that live within a self-secreted matrix of extracellular polymers. They exhibit high tolerance to antimicrobial agents, and experimental evidence indicates that in many instances repeated doses of antimicrobials further reduce disinfection efficiency due to an adaptive stress response. In this investigation, a mathematical model of bacterial adaptation is presented consisting of an adapted-unadapted population system embedded within a moving boundary problem coupled to a reaction-diffusion equation. The action of antimicrobials on biofilms under different dosing protocols is studied both analytically and numerically. We find the limiting behavior of solutions under periodic and on-off dosing as the period is made very large or very small. High dosages often carry undesirable side effects so we specially consider low dosing regimes. Our results indicate that on-off dosing for small doses of biocide is more effective than constant dosing. Moreover, in a specific case, on-off dosing for short periods is again more effective regardless of the biocide dose. We also provide sufficient conditions for the eradication of biofilms under a constant dosing regime.
\end{abstract}

Key words. biofilm, dosing strategies, analysis

AMS subject classifications. 92B, 35L02

DOI. $10.1137 / 080739070$

1. Introduction. Biofilms form when sufficient numbers of microorganisms adhere to surfaces in aqueous environments and encase themselves in a self-excreted slimy, glue-like matrix. It has been estimated that possibly $99 \%$ of bacteria live in biofilm communities rather than the free-living, planktonic state [26]. Biofilms have been found to be involved in a wide variety of microbial infections in the body, by one estimate $80 \%$ of all bacterial infections [28]. They are also ubiquitous in industrial and civilian contexts, including, for example, water distribution and treatment systems $[9,40]$. Thus, effective biofilm control agents are highly desirable. There are in fact many available antimicrobial substances that can effectively eradicate planktonic populations and so would seem to be likely control candidates. However, bacteria living in biofilms are notorious for their recalcitrance: minimum inhibitory concentrations (MICs) of antimicrobials for bacteria in biofilms can be on the order of $10^{3}$ times larger than MICs for the same organisms suspended in planktonic cultures; see, e.g., $[18,24]$. It is in fact believed that there are multiple resistance mechanisms present within a biofilm community, including weak antimicrobial penetration into the biofilm due to reaction-diffusion barriers and slow growth (many antimicrobials are less effective against inactive microbes) due to nutrient limitation [23, 24, 34]. Studies have shown an apparent failure of certain antimicrobial agents to penetrate the biofilm due

${ }^{*}$ Received by the editors October 27, 2008; accepted for publication (in revised form) September 13, 2010; published electronically November 11, 2010. This work was partially supported by NIH grant 5R01GM067245-02, EPSRC grant EP/F014589/1, and NSF grant O112050.

http://www.siam.org/journals/siap/70-8/73907.html

${ }^{\dagger}$ Mathematical Biosciences Institute, The Ohio State University, Columbus, OH 43210 (bszomolay @mbi.osu.edu).

${ }^{\ddagger}$ Department of Mathematical Sciences, Montana State University, Bozeman, MT 59717 (klapper @math.montana.edu).

$\S$ Maxwell Institute for Mathematical Sciences, The University of Edinburgh, Edinburgh, EH9 3JZ, United Kingdom (M.Dindos@ed.ac.uk). 
to being consumed by the matrix or binding to the biofilm components, and due to the degree of biofilm thickness (thin versus thick) [23].

The focus of this paper is on a specific stress response to antimicrobial stimulus designated adaptive resistance [34]. Adaptive resistance is not a true resistance since the MIC (of resuspended cells in the case of biofilms) is not altered. Instead, adaptive response might be a consequence of the action of antibiotics on bacteria, rather than the adaptation of bacteria themselves. However, this is the term that has been adopted in the literature. Thus by "adaptive" we do not refer to the widely familiar mechanism of selective emergence of a genetically distinct, specially resistant strain, but rather to a nonselective phenotypic response (i.e., changed pattern of gene expression) available to a general population when subject to an applied stress [41]. Such a response has been observed in planktonic microbial populations exposed to sublethal antimicrobial dosages; see, e.g., [29]. It is hypothesized in the case of biofilms that, even when exposed to what would be a lethal dosage for a planktonic population, shelter offered to organisms located deeper in the biofilm at least temporarily results in sublethal dosages of antimicrobial, thus allowing time for upregulation of adaptive defenses. Examples include induction of genes resulting in upregulation of catalase production in response to hydrogen peroxide [14] and upregulation of beta-lactamases in response to beta-lactam antibiotics [3, 15]. By the way, it is perhaps not surprising that microbes have such defenses in their arsenals as many antimicrobials are derived from the environment and hence not necessarily unfamiliar to their targets. Both of the antimicrobials just mentioned are found in natural environments, for example.

While in principal available to either planktonic- or biofilm-state organisms, in practice, for reasons described above, adaptive stress response would seem to be much more effective for biofilm than for planktonic communities. A modeling study of both the biofilm and planktonic culture cases indicated that, due to diffusion-reaction barriers, very large increases in dosages would be necessary to prevent adaptive response in thick biofilms, suggesting that adaptive response is considerably more dangerous in biofilms than in planktonic populations [39]. Thus we concentrate our attention here on biofilms. We note though that a thin biofilm lacks much of a matrix barrier and hence, within the scope of this paper at least, can be considered similar to a planktonic population.

The phenomenon of adaptive response is clearly relevant to dosing strategy. Constant high-dosage treatment, though effective [12], may be expensive, unpractical, or even dangerous. An episodal regime thus seems attractive in order to allow reversion to the unadapted state and to allow disinfected material to be cleared between dosages, reducing the reaction-diffusion barrier to antimicrobial access [36]. There is both in vivo and in vitro evidence to support this conjecture: Karlowsky, Zelenitsky, and Zhanel [19] have shown that the adaptive resistance of Gram-negative bacteria to aminoglycoside develops in immunocompromised patients and showed evidence that a treatment with MIC ratios and once-daily dosing results in the best outcome. This finding is also supported by [5]; dose administration at 24-hour intervals may increase efficacy by allowing time for adaptive resistance to reverse. The advantage of longer dosing intervals for aminoglycosides was studied earlier in [6]. Due to the high MICs required for biofilm control, it is plausible that, given a fixed quantity of antimicrobial and an unwelcome biofilm, a short, relatively high dosage application regime may be preferable to a prolonged, low dosage one. Available evidence is consistent with this hypothesis [17]. Nevertheless, even a very high dose is not sufficient for full disinfection of a thick biofilm if applied for only a short duration due to diffusive-reactive barriers [39]. Hence regrowth (which favors a dosing strategy with short off times or even 
constant dosing) is to be expected to eventually necessitate further treatment. On the other hand, adaptive responses come at an expense to individual microorganisms and hence can be expected to be downregulated at some point (which favors a dosing strategy with long off times) in the absence of antimicrobial antagonism. Development of a long-term dosage strategy which balances regrowth and downregulation time scales is hence desirable. Given a fixed average rate of antimicrobial application, is it better to apply a constant dose or a periodic one? If periodic, what should the period be? In between application of high dosages, is it most advantageous to set the dosage to zero in order to encourage downregulation of adaptive resistance? Note that, as already mentioned, one question we do not address concerns the effects of selective pressure on a special, resistant subpopulation; we suppose a single, clonal population throughout.

Choice of dosing schedule may be important. The study by Sanderson and Stewart [30] investigated the role of dosing protocols for the biocide monochloramine both mathematically and experimentally. Their model, which did not include any adaptive response mechanism, correctly simulated rapid disinfection followed by steady regrowth. However, it also predicted that, because of a reduction in the reactiondiffusion barrier, a second dose would be more effective than the first dose, whereas in actuality the opposite was observed in the experiments, due, presumably, to adaptive response and possibly short intervals between dosings. Adaptive response has been problematic in other dosing studies as well. For example, in [27] investigators observed that efficacy of twice daily, pulsed doses of chlorhexidine (a common active ingredient in mouthwash) applied to dental biofilms decreased dramatically after the first application, while, in [16], investigators found that insufficiently high dosages of antimicrobial in a heat exchange system actually seemed to enhance biofilm activity, maybe due to adaptive stimulation of production of extracellular matrix material. These examples and others suggest that if sustained high dosage attack is not practical, dosing strategies need to take into account the possibility of adaptive response defenses.

The objective of this study is to build on a previously introduced biofilm model $[39,37]$ in order to analyze different dosing protocols with particular focus on the contrast between constant and periodic or on-off dosing regimens. To distinguish between dosing strategies, we define $u(t)$ as the externally applied biocide concentration through the biofilm bulk-fluid interface. The term "periodic dosing" is used if $u(t)$ is periodic in time $t$ and positive on a set of full measure. The term "on-off dosing" is used if $u(t)$ is nonnegative periodic and zero on a set of positive measure. In this work, results concerning constant, periodic, and on-off dosing strategies against biofilms are presented.

As a final remark, we note the existence of another type of tolerance, namely, through the so-called persister cell [22]. Persisters are special cells present in microbial populations that, in contrast to adaptive responders [7], exhibit a remarkable general tolerance towards antimicrobials. Their nature is still a subject of investigation. It has been proposed that persisters are, essentially, normal cells that have temporarily changed phenotype into a protective state [20], though other possible explanations exist; see, e.g., [21, 2]. One phenotype-based theory posits that the transition to and from the persister state is mediated by the presence of an antimicrobial stress [22]. While biologically distinct from adaptive response defenses, this theory of persisters leads to models that are mathematically related to that presented here and also suggest utility of on-off type dosing regimes [10, 11, 32].

2. The biofilm model. Our model is an extension of a previously proposed one-dimensional moving boundary biofilm model that was linear in the variable $B$ (an- 
timicrobial concentration) [37]. Bacterial adaptation was analyzed, including growth and detachment. Global existence and other properties of solutions were shown and the corresponding steady states were studied. In particular, sufficient conditions for the existence of trivial/nontrivial steady states were established. Here we will replace the linear death rates due to antibiotic action by nonlinear functions $\varphi$ and $\psi$. When linear, these terms lead to the unrealistic conclusion that the most optimal dosing strategy is a delta distribution (i.e., a very large dose over a very small time period), which, in turn, results in qualitatively incorrect predictions. An appropriate choice of the functions $\varphi$ and $\psi$ (for example, bounded) removes this problem. This modification does not invalidate the existence results from [37], so the results for the existence of solutions of the PDE system and the corresponding steady states carry over.

We now describe the mathematical model of the dynamics of antimicrobial concentration, biomass constituents, and biofilm thickness. The bacterial population is composed of the following phenotypes: unadapted cells volume fraction $X_{u}$, adapted cells volume fraction $X_{a}$, dead unadapted cells volume fraction $X_{u d}$, and dead adapted cells volume fraction $X_{a d}$. Volume-specific densities are assumed constant. It is assumed for simplicity that there is no substrate limitation (saturation occurs) and that biomass phases can be regarded as incompressible. As cells alone do not fill the volume, it is sometimes supposed that $X_{u}+X_{u d}+X_{a}+X_{a d}=1-\epsilon$, where $\epsilon$ is the volume fraction of water and is constant in space and time. Since this fraction is constant, without affecting the results of this paper we can in fact set $\epsilon \equiv 0$. The equations of the cell volume fractions are then, on spatial domain $x \in[0, L(t)]$,

$$
\begin{aligned}
& \frac{\partial X_{u}}{\partial t}+\frac{\partial}{\partial x}\left(X_{u} v\right)=-\underbrace{\varphi(B) X_{u}}_{\text {death }}-\underbrace{\lambda(B) X_{u}}_{\text {adaptation }}+\underbrace{\alpha X_{u}}_{\text {growth }}+\gamma X_{a}, \\
& \frac{\partial X_{u d}}{\partial t}+\frac{\partial}{\partial x}\left(X_{u d} v\right)=\varphi(B) X_{u}, \\
& \frac{\partial X_{a}}{\partial t}+\frac{\partial}{\partial x}\left(X_{a} v\right)=\lambda(B) X_{u}-\underbrace{\psi(B) X_{a}}_{\text {death }}+\underbrace{\alpha X_{a}}_{\text {growth }}-\underbrace{\gamma X_{a}}_{\text {reversion }}, \\
& \frac{\partial X_{a d}}{\partial t}+\frac{\partial}{\partial x}\left(X_{a d} v\right)=\psi(B) X_{a},
\end{aligned}
$$

where $x=0$ represents the solid wall (substratum), $x=L$ represents the solvent interface, and the biofilm is uniform in the other, horizontal directions. In (1)-(4), $v$ is the velocity driving advective biomass transport and $B$ is the antimicrobial concentration. The population of unadapted cells in (1) changes due to growth, death from antibiotic action, loss from transition to adapted cells, and gain as the adapted cells revert back to unadapted cells. The population of adapted cells in (3) changes due to growth, death, and loss due to reversion. The dead cell populations in (2) and (4) increase by disinfection of living cells to dead ones. We assume that the unadapted cell population dies at a faster rate than adapted cells due to the adaptive response, i.e., $\varphi>\psi$. Distinguishing the two dead cell populations (rather than considering them as one) is for bookkeeping purposes only. Note that in the case of adaptation, reversion may be different from that of persisters, where reversion is sometimes assumed only in the absence of antibiotics (see [11]). We also assume that the growth rates of unadapted and adapted cells are the same. In actuality, presumably, unadapted cells grow faster, though the differences in growth rates may be small [33] and are unimportant here. This is in notable contrast to nongrowing or slowly growing persister cells [4]. 
We regard our model as dimensionless with time $t$ scaled by a typical experimental (or treatment) time $\tau$. It is assumed that living and dead cells react with the biocide at the same rate, i.e., cell-biocide reactions are independent of viability. That is, we suppose a reactive biocide. Nonreactive biocides are often similar in behavior because they typically require active targets, which in turn require an active limiting substrate, e.g., oxygen. If unadapted and adapted cell types react with biocide at the same rate, then the biocide concentration in dimensional variables is the solution of the equation

$$
\frac{\partial B}{\partial t}=D \frac{\partial^{2} B}{\partial x^{2}}-k B
$$

where $D$ is the diffusion rate and $k$ is the biocide-cell reaction rate. It is also assumed that in the biofilm the biocide concentration is quasi-static, i.e., the time derivative $\partial B / \partial t$ can be neglected. If $l$ is a characteristic biofilm thickness, then this assumption amounts to assuming that the characteristic time for diffusion $l^{2} D^{-1}$ is small compared to the duration of the biocide treatment $\tau$ and the other time scales present, including $1 / \alpha, 1 / \gamma, 1 / \lambda$, a usually reasonable assumption.

Biofilm thickness $L(t)$ changes in response to the advection of biomass and to detachment of biomass. In this model, detachment is treated as a surface erosion of the biofilm at a rate proportional to the square of the biofilm thickness, a standard practice for one-dimensional biofilm models [35]. Summing (1)-(4) and taking into account the incompressibility condition (total cell density is constant), we obtain a formula for the advection velocity $v$ given by (10) below. Altogether, the dimensionless model equations can be rewritten as

$$
\begin{aligned}
& \frac{\partial^{2} B}{\partial x^{2}}=\phi^{2} B \\
& \frac{\partial X_{u}}{\partial t}+v \frac{\partial X_{u}}{\partial x}=-(\varphi(B)+\lambda(B)) X_{u}+\alpha\left(1-X_{u}-X_{a}\right) X_{u}+\gamma X_{a}, \\
& \frac{\partial X_{u d}}{\partial t}+v \frac{\partial X_{u d}}{\partial x}=\varphi(B) X_{u}-\alpha\left(X_{u}+X_{a}\right) X_{u d}, \\
& \frac{\partial X_{a}}{\partial t}+v \frac{\partial X_{a}}{\partial x}=\lambda(B) X_{u}-\psi(B) X_{a}+\alpha\left(1-X_{u}-X_{a}\right) X_{a}-\gamma X_{a}, \\
& \frac{\partial X_{a d}}{\partial t}+v \frac{\partial X_{a d}}{\partial x}=\psi(B) X_{a}-\alpha\left(X_{u}+X_{a}\right) X_{a d}, \\
& \frac{\partial v}{\partial x}=\alpha\left(X_{u}+X_{a}\right) \\
& \frac{d L}{d t}=v(L, t)-\sigma L^{2}
\end{aligned}
$$

again with $x \in[0, L(t)]$. We use the same symbols as before, although they are now scaled. Also, we would like to point out that the detachment term $\sigma L^{2}$ could be replaced by any reasonable nonnegative continuous function $f(L)$ as long as $f$ grows faster than linearly for sufficiently large $L$.

The parameter $\phi^{2}$, square of the so-called Thiele modulus, is the ratio of the biocide-cell reaction time $\left(k X_{0}\right)^{-1}$ and the characteristic time of diffusion $l^{2} D^{-1}$ (with system length scale $l$ ); it is an indicator of effectiveness of the biocide against the biofilm. In particular, over times short compared to growth and erosion time scales, biofilm thickness is almost constant with, say, approximate thickness $l$. Since $\sqrt{D / k X_{0}}$ has units of length, then $1 / \phi=\sqrt{D / k X_{0}} / l$ is approximately the depth in scaled units of the biofilm layer in which most disinfection occurs (see [39]). 
The boundary conditions are

$$
\begin{aligned}
& \frac{\partial B}{\partial x}(0, t)=0, \quad B(L(t), t)=u(t) \quad \text { for } \quad 0 \leq t \leq P, \\
& v(0, t)=0 \quad \text { for } \quad 0 \leq t \leq P
\end{aligned}
$$

where $u(t)$, the externally applied antimicrobial concentration, is a nonnegative piecewise continuous periodic function of period $P$. It follows directly from (5) and (12) that the biocide concentration is given by

$$
B(x, t)=u(t) \frac{\cosh (\phi x)}{\cosh (\phi L(t))} .
$$

The function $\lambda(B)$ is defined as

$$
\lambda(B)= \begin{cases}\lambda & \text { if } B>0 \\ 0 & \text { if } B=0\end{cases}
$$

Note thus that adaptation occurs only in the presence of biocide. The disinfection rates $\varphi$ and $\psi$ are monotone increasing, nonnegative, and locally Lipschitz with at most polynomial growth such that $\varphi(0)=\psi(0)=0$ (the linear case was previously studied in [37]). A list of the model parameters is given in Table 1.

TABLE 1

Model parameters.

\begin{tabular}{|c|l|}
\hline$\phi$ & Thiele modulus \\
\hline$\alpha$ & maximum specific growth rate \\
\hline$\gamma$ & adapted-unadapted cell reversion rate \\
\hline$\lambda(B)$ & unadapted-adapted cell transformation rate \\
\hline$\varphi(B)$ & biocide-unadapted cell reaction rate \\
\hline$\psi(B)$ & biocide-adapted cell reaction rate \\
\hline$\sigma$ & detachment coefficient \\
\hline
\end{tabular}

In order to explore optimal dosing strategies, we introduce functionals to be optimally minimized with respect to the externally applied antimicrobial concentration $u(t)$. In general, antimicrobials can be bactericidal (killing bacteria, e.g., penicillin) and bacteriostatic (inhibiting the growth of bacteria, e.g., tetracycline). Motivated by these two classifications, we introduce two types of minimizing functionals: functional $J$ (the long-term average of the number of viable organisms roughly speaking) and functional $J_{L}$ (the long-term average of biofilm thickness), i.e.,

$$
J(u)=\lim _{T \rightarrow \infty} \frac{1}{T} \int_{0}^{T} \int_{0}^{L(t)}\left(X_{u}(x, t)+X_{a}(x, t)\right) d x d t
$$

and

$$
J_{L}(u)=\lim _{T \rightarrow \infty} \frac{1}{T} \int_{0}^{T} L(t) d t .
$$

Throughout this paper we will assume, when $u(t)$ is periodic, that the system (5)-(11) has a periodic solution satisfying the boundary conditions (12). In fact, numerical simulations suggest that given any initial data, the solution of the system (5)-(11) 
becomes periodic as $t \rightarrow \infty$ with length of period $P$. This implies that formulas (14) and (15) simplify to

$$
J(u)=\lim _{T \rightarrow \infty} \frac{1}{P} \int_{T}^{T+P} \int_{0}^{L(t)}\left(X_{u}(x, t)+X_{a}(x, t)\right) d x d t
$$

and

$$
J_{L}(u)=\lim _{T \rightarrow \infty} \frac{1}{P} \int_{T}^{T+P} L(t) d t .
$$

This form of the functionals is numerically more convenient and will be used throughout.

3. Analysis of dosing protocols. We consider three dosing types: constant dosing, periodic dosing, and on-off dosing (as defined in the introduction). The goal is to find a dosing strategy for which the functionals defined above are minimal. We start with constant dosing by reviewing and generalizing the results from [37]. The other two dosing strategies will be discussed later making use of and extending results obtained for constant dosing.

3.1. Constant dosing. The existence of steady-state solutions for the biofilm model with functions $\varphi$ and $\psi$ linear has been established in [37]. However, all the results from [37] remain true even for the more general functions $\varphi$ and $\psi$ we allow here. Denote by $(S)$ the system of steady-state equations corresponding to (5)-(10) with boundary conditions

$$
\frac{\partial B}{\partial x}(0)=0, \quad B(L)=u_{0}, \quad v(0)=0, \quad v(L)=\sigma L^{2} .
$$

For any $u_{0} \geq 0$ there exists at least one solution (possibly the trivial one $L=0$ ) of the system $(S)$. In fact, we are able to state sufficient conditions for the existence of trivial/nontrivial steady states depending on dosage $u_{0}$ as formulated in Theorem 1 . The statement formulated below holds not only for the biologically relevant case $\varphi>\psi$, but for any positive parameters $\varphi, \psi$. More importantly, it provides sufficient and necessary conditions for the case when the biofilm cannot be eradicated, or, otherwise, it gives an approximate lower estimate for the biocide dose that is necessary for eradication.

THEOREM 1. Given parameters $\alpha, \gamma, \sigma, \lambda$ and functions $\varphi, \psi$ which are monotone increasing, nonnegative, and locally Lipschitz with at most polynomial growth such that $\varphi(0)=\psi(0)=0$, there exists a number $u_{\max }, 0<u_{\max } \leq \infty$, such that for any $u_{0} \in\left[0, u_{\max }\right)$ there is at least one continuous nontrivial solution of the system $(S)$ satisfying the boundary conditions (18). Let $M_{\varphi}=\lim _{u \rightarrow \infty} \varphi(u)$ and $M_{\psi}=\lim _{u \rightarrow \infty} \psi(u)$. Then $u_{\max }=\infty$ if and only if one of these holds:

(i) $M_{\varphi}<\infty, M_{\psi}=\infty$, and $M_{\varphi}+\lambda \leq \alpha$;

(ii) $M_{\varphi}=\infty, M_{\psi}<\infty$, and $M_{\psi}+\gamma \leq \alpha$;

(iii) $M_{\varphi}=M_{\psi}=\infty$ and $\gamma \leq \alpha$;

(iv) $M_{\varphi}<\infty, M_{\psi}<\infty$, and $\Gamma_{\infty} \leq \alpha$, where

$$
\Gamma_{\infty}=\frac{M_{\psi}+\gamma+M_{\varphi}+\lambda-\sqrt{\left(M_{\psi}+\gamma+M_{\varphi}+\lambda\right)^{2}-4\left(M_{\psi} M_{\varphi}+\lambda M_{\psi}+\gamma M_{\varphi}\right)}}{2} .
$$


Otherwise, $u_{\max }$ is a positive number with lower bound $u_{\max } \geq \bar{u}$, where $\bar{u}$ is the smallest positive solution of an implicit equation

$$
\alpha=\frac{\psi(\bar{u})+\gamma+\varphi(\bar{u})+\lambda-\sqrt{\mathcal{F}(\bar{u})}}{2},
$$

with $\mathcal{F}(\bar{u})=(\psi(\bar{u})+\gamma+\varphi(\bar{u})+\lambda)^{2}-4(\psi(\bar{u}) \varphi(\bar{u})+\lambda \psi(\bar{u})+\gamma \varphi(\bar{u}))$.

Remark. Note that the theorem statement does not discuss nonuniqueness of nontrivial steady-state solutions. It has been shown in [37] that these solutions are unique for small doses of biocide $u_{0}$, but for larger doses nonuniqueness in fact can occur. We remark here that the biologically most plausible scenarios for failure to kill the biofilm are (ii) and (iv). It is easy to see that $\Gamma_{\infty} \rightarrow M_{\psi}+\gamma$ as $M_{\phi} \rightarrow \infty$. Hence, (ii) follows from (iv). We conclude that if the adapted cell killing efficiency is small $\left(M_{\psi} \ll 1\right)$ and the adapted cells grow faster than they revert to the unadapted state $(\gamma<\alpha)$, even a significant increase in the unadapted cell killing efficiency $\left(M_{\phi} \gg 1\right)$ is not enough to guarantee eradication of the biofilm. Such, for example, may be the case in [1], where none of the antibiotics studied was able to completely eradicate the biofilm. Growth rate appears to be one of the main factors in mediating recalcitrance of biofilms to antimicrobial agents.

Proof. This theorem was proved for the case of linear functions $\varphi$ and $\psi$ in [37]. Based on an existence result from [37], the system $(S)$ has a nontrivial continuous solution of length $L$, provided that the autonomous system

$$
\begin{aligned}
& \frac{d x_{u}}{d t}=-\left(\varphi\left(B_{0}\right)+\lambda\right) x_{u}+\alpha\left(1-x_{u}-x_{a}\right) x_{u}+\gamma x_{a}, \\
& \frac{d x_{a}}{d t}=\lambda x_{u}-\psi\left(B_{0}\right) x_{a}+\alpha\left(1-x_{u}-x_{a}\right) x_{a}-\gamma x_{a}
\end{aligned}
$$

has a positive $\left(x_{u}, x_{a}>0\right)$ equilibrium. Here $B_{0}=\frac{u_{0}}{\cosh \phi L}$. Analysis of this autonomous system as done in [37] implies that such an equilibrium exists if and only if

$$
\alpha>\frac{\psi\left(B_{0}\right)+\gamma+\varphi\left(B_{0}\right)+\lambda-\sqrt{\mathcal{F}\left(B_{0}\right)}}{2},
$$

where $\mathcal{F}$ is the function defined above. If $u_{0}$ is small, then so is $B_{0}$, as $B_{0} \leq u_{0}$. It follows that for small enough values of $u_{0}$ the condition (20) is always satisfied as the expression on the right-hand side is close to zero. From this observation, the existence of nontrivial continuous steady-state solutions follows for $u_{0}$ on some interval $\left[0, u_{\max }\right)$.

Further examination of condition (20) implies that it will always be satisfied (for arbitrary large $u_{0}$ ) in any of the three cases stated in Theorem 1 . Otherwise, the condition yields at least the implicit lower bound $\bar{u}$ on the value of $u_{\max }$, and if $u_{0}<\bar{u}$, then the required condition (20) holds.

3.2. Periodic and on-off dosing. In this section we consider time varying dosing strategies. Of particular interest is the special case we call on-off dosing, where a dose is applied to the biofilm for a fraction of overall time only and no dose is given for the rest of the period. The idea is to allow a "recovery time" for the biofilm. The presence of biocide causes biofilm to become tolerant, a state manifested through increased proportion $X_{a}$ of adapted cells. To counter, we decrease time of presence of biocide which should (and will) lower the formation of adapted cells. This dosing strategy has the potential to be more effective overall than constant dosing. 
We introduce the following notation. Let $u$ be a nonnegative, periodic, continuous (or, more generally, piecewise continuous) function with period 1. For any positive number $\omega>0$ we define the function

$$
u^{\omega}(t)=u(\omega t) \quad \text { for } t \in \mathbb{R} .
$$

Note that $u^{\omega}$ is a periodic function of period $P=1 / \omega$ and, hence, $\omega$ is its frequency. Denote by $B^{\omega}, X_{u}^{\omega}, X_{u d}^{\omega}, X_{a}^{\omega}, X_{a d}^{\omega}, v^{\omega}, L^{\omega}$ the corresponding periodic solution of the system (5)-(11) with boundary conditions (12). We denote the values of the corresponding functionals by $J^{\omega}$ and $J_{L}^{\omega}$, respectively. Let $\mu$ be the Lebesgue measure of the set $\{t \in[0,1] ; u(t)>0\}$. It follows that $\mu \in(0,1]$. In the case of on-off dosing, where $u(t)$ is a step function such that $u(t)>0$ on $[0, \mu]$ and $u(t)=0$ on $(\mu, 1]$, the parameter $\mu$ is the ratio of the dosing time to the period. From now on we will use the term "dosing ratio" for the variable $\mu$. Note that $\mu=1$ corresponds to constant dosing, and the case $\mu=0$ is not defined.

It turns out that we can analyze the limiting behavior of periodic solutions for large and small frequencies. More precisely, in the theorems below we make statements about the limit of the functionals $J^{\omega}, J_{L}^{\omega}$ as the frequency $\omega \rightarrow \infty$ and $\omega \rightarrow 0+$. We start with a result relevant to the high frequency case.

TheOREM 2. Let $u_{0}=\int_{0}^{1} u(t) d t$. Then any increasing sequence $\omega_{1}, \omega_{2}, \omega_{3}, \ldots$ such that $\omega_{n} \rightarrow \infty$ has a subsequence $\left(\omega_{n_{i}}\right)_{i \in N}$ satisfying

$$
L^{\infty}=\lim _{i \rightarrow \infty} L^{\omega_{n_{i}}}(t) \quad \text { uniformly for all } t>0,
$$

and all functions $B^{\omega_{n_{i}}}(x, t), X_{a}^{\omega_{n_{i}}}(x, t), X_{u}^{\omega_{n_{i}}}(x, t), X_{a d}^{\omega_{n_{i}}}(x, t), X_{u d}^{\omega_{n_{i}}}(x, t), v^{\omega_{n_{i}}}(x, t)$ converge for $x \in\left[0, L^{\infty}\right)$ and $t>0$ to functions $B^{\infty}(x), X_{a}^{\infty}(x), X_{u}^{\infty}(x), X_{a d}^{\infty}(x)$, $X_{u d}^{\infty}(x), v^{\infty}(x)$ that are independent of $t>0$. In the case of function $B^{\infty}$ the convergence is only in the weak sense; all other functions converge uniformly in $x$.

Moreover, these functions satisfy on the interval $\left[0, L^{\infty}\right]$ the equations of a steadystate solution

$$
\begin{aligned}
& \frac{\partial^{2} B^{\infty}}{\partial x^{2}}=\phi^{2} B^{\infty}, \\
& v^{\infty} \frac{\partial X_{u}^{\infty}}{\partial x}=-\left(\Phi\left(B^{\infty}\right)+\mu \lambda\right) X_{u}^{\infty}+\alpha\left(1-X_{u}^{\infty}-X_{a}^{\infty}\right) X_{u}^{\infty}+\gamma X_{a}^{\infty}, \\
& v^{\infty} \frac{\partial X_{u d}^{\infty}}{\partial x}=\Phi\left(B^{\infty}\right) X_{u}^{\infty}-\alpha\left(X_{u}^{\infty}+X_{a}^{\infty}\right) X_{u d}^{\infty}, \\
& v^{\infty} \frac{\partial X_{a}^{\infty}}{\partial x}=\mu \lambda X_{u}^{\infty}-\Psi\left(B^{\infty}\right) X_{a}^{\infty}+\alpha\left(1-X_{u}^{\infty}-X_{a}^{\infty}\right) X_{a}^{\infty}-\gamma X_{a}^{\infty}, \\
& v^{\infty} \frac{\partial X_{a d}^{\infty}}{\partial x}=\Psi\left(B^{\infty}\right) X_{a}^{\infty}-\alpha\left(X_{u}^{\infty}+X_{a}^{\infty}\right) X_{a d}^{\infty}, \\
& \frac{\partial v^{\infty}}{\partial x}=\alpha\left(X_{u}^{\infty}+X_{a}^{\infty}\right), \\
& v^{\infty}\left(L^{\infty}, .\right)=\sigma\left(L^{\infty}\right)^{2},
\end{aligned}
$$

with the boundary condition $B^{\infty}\left(L^{\infty}\right)=u_{0}$. Here the functions $\Phi$ and $\Psi$ are defined by

$$
\Phi(z)=\int_{0}^{1} \varphi\left(\frac{u(t)}{u_{0}} z\right) d t \quad \text { and } \quad \Psi(z)=\int_{0}^{1} \psi\left(\frac{u(t)}{u_{0}} z\right) d t
$$

Copyright $@$ by SIAM. Unauthorized reproduction of this article is prohibited. 
Finally, values of the functionals $J^{\omega_{n_{i}}}$ and $J_{L}^{\omega_{n_{i}}}$ converge to

$$
J^{\infty}=\lim _{i \rightarrow \infty} J^{\omega_{n_{i}}}=\int_{0}^{L^{\infty}}\left(X_{a}^{\infty}+X_{u}^{\infty}\right) d x, \quad J_{L}^{\infty}=\lim _{i \rightarrow \infty} J_{L}^{\omega_{n_{i}}}=L^{\infty} .
$$

Remark. Note that if $\varphi$ is linear, then $\varphi=\Phi$, and the same is also true for $\psi$. It follows that $\Phi$ and $\Psi$ are locally Lipschitz, increasing functions with $\Phi(0)=\Psi(0)=0$. For the choice of on-off dosing that we make below, the function $u(t)$ is defined as $u_{0} / \mu$ on the interval $[0, \mu]$ and zero on $(\mu, 1]$. Hence, $\Phi(z)=\mu \varphi(z / \mu)$ and $\Psi(z)=\mu \psi(z / \mu)$.

Proof. All functions $L^{\omega_{n}}$ are uniformly bounded from above by $\frac{\alpha}{\sigma}$ (cf. Lemma 2 of [37]). Indeed, if $L(t)>\frac{\alpha}{\sigma}$, then

$$
\frac{d L}{d t}=v(L)-\sigma L^{2}<\alpha L-\sigma \frac{\alpha}{\sigma} L=0,
$$

which implies that for sufficiently large $t$ we have $L(t) \leq \frac{\alpha}{\sigma}$. In our case, we consider periodic solutions. If $L(0)>\frac{\alpha}{\sigma}$, it would follow that $L(P)<L(0)$ (where $P$ is the period of our solution), which is a contradiction. Hence, $L(t) \leq \frac{\alpha}{\sigma}$ for all $t \geq 0$.

We can also obtain a uniform bound on $\frac{d L^{\omega_{n}}}{d t}$. Equation (11) implies

$$
-\frac{\alpha^{2}}{\sigma} \leq-\sigma\left(L^{\omega_{n}}(t)\right)^{2} \leq \frac{d L^{\omega_{n}}}{d t}=v\left(L^{\omega_{n}}\right)-\sigma\left(L^{\omega_{n}}\right)^{2} \leq v\left(L^{\omega_{n}}\right) \leq \alpha L^{\omega_{n}}(t) \leq \frac{\alpha^{2}}{\sigma} .
$$

Hence the functions $L^{\omega_{n}}$ are uniformly Lipschitz in $t$, i.e., also equicontinuous. By the Ascoli theorem there exists a subsequence $\left(n_{i}\right)$ such that the sequence of functions $L^{\omega_{n_{i}}}$ is convergent. Since the length of the period goes to zero, the limit must be a constant function, which we call $L^{\infty}$. If this limit is zero, the proof is complete-in this case $L^{\infty}=0$, which corresponds to the trivial steady-state solution.

The alternative case is more interesting. It follows from above that $L^{\infty}$ is constant in $t$. Since the functions $B^{\omega_{n_{i}}}$ are explicitly determined by $L^{\omega_{n_{i}}}$, we have that

$$
\frac{\cosh (\phi x)}{\cosh \left(\phi L^{\omega_{n_{i}}}(t)\right)} \rightarrow \frac{\cosh (\phi x)}{\cosh \left(\phi L^{\infty}\right)} \quad \text { uniformly in } x
$$

and

$$
u^{\omega_{n_{i}}}(t) \rightarrow u_{0} \quad \text { weakly, i.e., for any } \psi \in C_{0}^{\infty}(R): \quad \int u^{\omega_{n_{i}}}(t) \psi(t) d t \rightarrow u_{0} \int \psi(t) d t
$$

as $\omega_{n_{i}} \rightarrow \infty$. This follows immediately from the fact that $u_{0}$ is the average value of each function $u^{\omega}$. From this the product function $B^{\omega_{n_{i}}}(x, t)=u(t) \frac{\cosh (\phi x)}{\cosh \left(\phi L^{\omega n_{i}}(t)\right)}$ will converge weakly to $B^{\infty}(x)=u_{0} \frac{\cosh (\phi x)}{\cosh \left(\phi L^{\infty}\right)}$, where the weak convergence is understood in $t$, as defined above.

Now for each $\omega_{n_{i}}$ let us introduce new variables, which we denote by $\widetilde{B}^{i}, \widetilde{v}^{i}, \widetilde{X}_{u}^{i}$, $\widetilde{X}_{a}^{i}, \widetilde{X}_{u d}^{i}, \widetilde{X}_{a d}^{i}$, that will be functions only of variable $x$ and not of $t$. We will define them on the interval $\left[0, \widetilde{L^{i}}\right]$, where

$$
\widetilde{L^{i}}=\inf _{t} L^{\omega_{n_{i}}}(t)
$$

It follows immediately that $\widetilde{L^{i}} \rightarrow L^{\infty}$ as $i \rightarrow \infty$. The mentioned functions are defined 
via averaging over the period, which for index $i$ is $\omega_{n_{i}}^{-1}$, i.e.,

$$
\begin{aligned}
& \widetilde{X}_{a}^{i}(x)=\omega_{n_{i}} \int_{0}^{1 / \omega_{n_{i}}} X_{a}^{\omega_{n_{i}}}(x, t) d t, \quad \widetilde{X}_{a d}^{i}(x)=\omega_{n_{i}} \int_{0}^{1 / \omega_{n_{i}}} X_{a d}^{\omega_{n_{i}}}(x, t) d t, \\
& \widetilde{X}_{u}^{i}(x)=\omega_{n_{i}} \int_{0}^{1 / \omega_{n_{i}}} X_{u}^{\omega_{n_{i}}}(x, t) d t, \quad \widetilde{X}_{u d}^{i}(x)=\omega_{n_{i}} \int_{0}^{1 / \omega_{n_{i}}} X_{u d}^{\omega_{n_{i}}}(x, t) d t, \\
& \widetilde{B}^{i}(x)=\omega_{n_{i}} \int_{0}^{1 / \omega_{n_{i}}} B^{\omega_{n_{i}}}(x, t) d t, \widetilde{v}^{i}(x)=\omega_{n_{i}} \int_{0}^{1 / \omega_{n_{i}}} v^{\omega_{n_{i}}}(x, t) d t .
\end{aligned}
$$

To obtain equations that will be satisfied by these new functions, we will integrate (5)-(10) over the interval $\left[0, \omega_{n_{i}}^{-1}\right]$ and average (i.e., multiply by $\left.\omega_{n_{i}}\right)$. We have

$$
\begin{aligned}
& \widetilde{B}^{i}(x)=\omega_{n_{i}} \int_{0}^{1 / \omega_{n_{i}}} u(t) \frac{\cosh (\phi x)}{\cosh \left[\phi L^{\omega_{n}}(t)\right]} d t=u_{0} \frac{\cosh (\phi x)}{\cosh \left[\phi \widetilde{L}^{i}\right]}+e_{1}, \\
& \widetilde{v}^{i} \frac{\partial \widetilde{X}_{u}^{i}}{\partial x}=-\left(\Phi\left(\widetilde{B}^{i}\right)+\mu \lambda\right) \widetilde{X}_{u}^{i}+\alpha\left(1-\widetilde{X}_{u}^{i}-\widetilde{X}_{a}^{i}\right) \widetilde{X}_{u}^{i}+\gamma \widetilde{X}_{a}^{i}+e_{2}, \\
& \widetilde{v}^{i} \frac{\partial \widetilde{X}_{u d}^{i}}{\partial x}=\Phi\left(\widetilde{B}^{i}\right) \widetilde{X}_{u}^{i}-\alpha\left(\widetilde{X}_{u}^{i}+\widetilde{X}_{a}^{i}\right) \widetilde{X}_{u d}^{i}+e_{3}, \\
& \widetilde{v}^{i} \frac{\partial \widetilde{X}_{a}^{i}}{\partial x}=\mu \lambda \widetilde{X}_{u}^{i}-\Psi\left(\widetilde{B}^{i}\right) \widetilde{X}_{a}^{i}+\alpha\left(1-\widetilde{X}_{u}^{i}-\widetilde{X}_{a}^{i}\right) \widetilde{X}_{a}^{i}-\gamma \widetilde{X}_{a}^{i}+e_{4}, \\
& \widetilde{v}^{i} \frac{\partial \widetilde{X}_{a d}^{i}}{\partial x}=\Psi\left(\widetilde{B}^{i}\right) \widetilde{X}_{a}^{i}-\alpha\left(\widetilde{X}_{u}^{i}+\widetilde{X}_{a}^{i}\right) \widetilde{X}_{a d}^{i}+e_{5}, \\
& \frac{\partial \widetilde{v}^{i}}{\partial x}=\alpha\left(\widetilde{X}_{u}^{i}+\widetilde{X}_{a}^{i}\right), \\
& 0=v\left(\widetilde{L}^{i}, .\right)-\sigma\left[\widetilde{L}^{i}\right]^{2}+e_{6} .
\end{aligned}
$$

Here $e_{1}, e_{2}, \ldots, e_{6}$ are the error terms that arise from nonlinear terms. We do not write all of them explicitly, but as an example we look in detail at

$$
\begin{aligned}
\omega_{n_{i}} \int_{0}^{1 / \omega_{n_{i}}} v^{\omega_{n_{i}}} \frac{\partial X_{u}^{\omega_{n_{i}}}}{\partial x} d t & =\omega_{n_{i}} \int_{0}^{1 / \omega_{n_{i}}} \widetilde{v}^{i} \frac{\partial X_{u}^{\omega_{n_{i}}}}{\partial x} d t+\omega_{n_{i}} \int_{0}^{1 / \omega_{n_{i}}}\left(v^{\omega_{n_{i}}}-\widetilde{v}^{i}\right) \frac{\partial X_{u}^{\omega_{n_{i}}}}{\partial x} d t \\
& =\widetilde{v}^{i} \frac{\partial \widetilde{X}_{u}^{i}}{\partial x}+e
\end{aligned}
$$

where $|e| \leq\left\|\widetilde{v}^{i}-v^{\omega_{n_{i}}}\right\|_{\infty}\left\|\frac{\partial \widetilde{X}_{u}^{\omega_{n}}}{\partial x}\right\|_{\infty}$.

We also look at the origin of the functions $\Phi$ and $\Psi$. Consider, for example, the term $\varphi\left(B^{\omega_{n_{i}}}\right) X_{u}^{\omega_{n_{i}}}$ in (6). After averaging we obtain

$$
\omega_{n_{i}} \int_{0}^{1 / \omega_{n_{i}}} \varphi\left(B^{\omega_{n_{i}}}\right) X_{u}^{\omega_{n_{i}}} d t=\omega_{n_{i}} \int_{0}^{1 / \omega_{n_{i}}} \varphi\left(B^{\omega_{n_{i}}}\right) \widetilde{X}_{u}^{i} d t+e^{\prime},
$$

where

$$
\left|e^{\prime}\right|=\omega_{n_{i}}\left|\int_{0}^{1 / \omega_{n_{i}}} \varphi\left(B^{\omega_{n_{i}}}\right)\left(X_{u}^{\omega_{n_{i}}}-\widetilde{X}_{u}^{i}\right) d t\right| \leq C\left(\varphi\left(\| B^{\omega_{n_{i}}}\right) \|_{\infty}\right)\left\|\widetilde{X}_{u}^{i}-X_{u}^{\omega_{n_{i}}}\right\|_{\infty} .
$$

Working further with (39) we see that

$$
\omega_{n_{i}} \int_{0}^{1 / \omega_{n_{i}}} \varphi\left(B^{\omega_{n_{i}}}\right) \widetilde{X}_{u}^{i} d t=\widetilde{X}_{u}^{i} \omega_{n_{i}} \int_{0}^{1 / \omega_{n_{i}}} \varphi\left(u^{\omega_{n_{i}}}(t) \frac{\cosh (\phi x)}{\cosh \left(\phi \widetilde{L}^{i}\right)}\right) d t+e^{\prime \prime},
$$

Copyright $@$ by SIAM. Unauthorized reproduction of this article is prohibited. 
where

$$
\left|e^{\prime \prime}\right|=\omega_{n_{i}}\left|\widetilde{X}_{u}^{i}\right|\left|\int_{0}^{1 / \omega_{n_{i}}}\left(\varphi\left(u^{\omega_{n_{i}}}(t) \frac{\cosh (\phi x)}{\cosh \left(\phi L^{\omega_{n_{i}}}\right)}\right)-\varphi\left(u^{\omega_{n_{i}}}(t) \frac{\cosh (\phi x)}{\cosh \left(\phi \widetilde{L}^{i}\right)}\right)\right) d t\right| .
$$

The fact that $\varphi$ is Lipschitz allows a bound $\left|e^{\prime \prime}\right| \leq C\left\|L^{\omega_{n_{i}}}-\widetilde{L}^{i}\right\|_{\infty}$. After change of variables the first term of (40) becomes

$$
\widetilde{X}_{u}^{i} \int_{0}^{1} \varphi\left(u(t) \frac{\cosh (\phi x)}{\cosh \left(\phi \widetilde{L}^{i}\right)}\right) d t=\widetilde{X}_{u}^{i} \Phi\left(u_{0} \frac{\cosh (\phi x)}{\cosh \left(\phi \widetilde{L}^{i}\right)}\right)=\widetilde{X}_{u}^{i} \Phi\left(\widetilde{B}^{i}(x)\right) .
$$

Similar estimates are worked out for all other nonlinear terms. In total we obtain

$$
\begin{aligned}
\left|e_{1}\right|+\cdots+\left|e_{6}\right| & \leq C\left\|\widetilde{L}^{i}-L^{\omega_{n_{i}}}\right\|_{\infty}+C\left\|\widetilde{v}^{i}-v^{\omega_{n_{i}}}\right\|_{\infty}\left\|\frac{\partial \tilde{X}^{i}}{\partial x}\right\|_{\infty} \\
& +C\left(\|B\|_{\infty}\right)\left\|\widetilde{X}^{i}-X^{\omega_{n_{i}}}\right\|_{\infty} .
\end{aligned}
$$

Here $\tilde{X}^{i}$ represents all variables $\tilde{X}_{a}^{i}, \tilde{X}_{u}^{i}$, etc. Let us also remark that the terms $\mu \lambda$ in (33) and (35) occur because the original equations contain $\lambda$ only when $u(t)>0$, which happens on a set of size $\mu$.

What follows relies on the following technical lemma.

LEMMA 1. There exists a constant $K>0$ depending only on constants $\alpha, \phi, \lambda, \gamma, \sigma$, and $\|u\|_{\infty}$ such that the periodic solutions of (5)-(11) are Lipschitz continuous in variable $x$ with Lipschitz constant at most $K$, that is,

$$
\max \left\{\left\|\frac{\partial X_{a}}{\partial x}\right\|_{\infty},\left\|\frac{\partial X_{a d}}{\partial x}\right\|_{\infty},\left\|\frac{\partial X_{u}}{\partial x}\right\|_{\infty},\left\|\frac{\partial X_{u d}}{\partial x}\right\|_{\infty}\right\} \leq K .
$$

The crucial part of the claim in this lemma is that the bound on the Lipschitz norm is uniform and depends only on the constants and $L^{\infty}$ norm of $u$. We will not include the proof; this lemma is closely connected with the question of existence of periodic solutions for which we also did not include the proof. However, the argument would be the same as a similar proof that establishes that the steady-state solution is also Lipschitz in $x$. We did not present it explicitly, as it was not necessary, but in the last part of the proof of the existence of the steady-state solution we derived an estimate on the distance $\rho$ of two steady-state solutions in the form $\frac{d \rho}{d x} \leq F(\rho)$. A slight modification of this estimate provides a uniform bound on the $\frac{d}{d x}$ derivative of the solution, which implies that the function is Lipschitz.

To return to our proof, once we have these estimates, we use (6)-(9) to conclude that for some $M$ independent of $i$ we also have that

$$
\max \left\{\left\|\frac{\partial X_{a}^{\omega_{n_{i}}}}{\partial t}\right\|_{\infty},\left\|\frac{\partial X_{a d}^{\omega_{n_{i}}}}{\partial t}\right\|_{\infty},\left\|\frac{\partial X_{u}^{\omega_{n_{i}}}}{\partial t}\right\|_{\infty},\left\|\frac{\partial X_{u d}^{\omega_{n_{i}}}}{\partial t}\right\|_{\infty}\right\} \leq M .
$$

Using the fact that functions $X_{a}^{\omega}, X_{u}^{\omega}, X_{a d}^{\omega}$, and $X_{u d}^{\omega}$ are periodic in $t$ with period $\omega_{n_{i}}^{-1}$, we then conclude that the oscillation (difference between sup and inf values) of these four functions is at most $M \omega_{n_{i}}^{-1} \rightarrow 0$ as $i \rightarrow \infty$. It follows from (42) that

$$
\left|e_{1}\right|+\cdots+\left|e_{5}\right| \leq C \omega_{n_{i}}^{-1}
$$

where $C$ is a constant depending only on the parameters of the equation and $\|u\|_{\infty}$. 
Now we choose a subsequence a second time. Equations (33), (35), and (37) appear exactly like those equations for a steady-state solution we studied closely before, plus some error terms. As we have shown, the solutions of these equation are all uniformly bounded and equicontinuous (Lemma 1), and hence by the Ascoli theorem there is a convergent subsequence as $i \rightarrow \infty$. On the other hand, as $i \rightarrow \infty$ the error terms vanish, and hence the limit of this subsequence satisfies exactly the equations for the steady-state solution. Let us call this limit $X_{u}^{\infty}, X_{a}^{\infty}, v^{\infty}$. The domain of these functions is the interval $\left[0, L^{\infty}\right)$.

So far we have uniform convergence of $\widetilde{X}_{u}^{i_{j}}$ to $X_{u}^{\infty}$, of $\widetilde{X}_{a}^{i_{j}}$ to $X_{a}^{\infty}$, and of $\widetilde{v}^{i_{j}}$ to $v^{\infty}$, where $\left(i_{j}\right)_{j \in N}$ is the selected subsequence. Recalling (44), we can drop the tilde and also see that $X_{u}^{\omega_{n_{i_{j}}}}(x, t)$ converges to $X_{u}^{\infty}(x)$ uniformly for arbitrary $t$ (and similarly for other functions). This is the desired subsequence.

Remark. It is possible that two different subsequences converge to two different limits. This happens only though if there is nonuniqueness of steady-state solutions. Otherwise, the limit is necessarily unique.

Now we have a look at the other limit $\omega \rightarrow 0+$, i.e., dosing time scale long compared to other time scales in the problem. In this case the length of period goes to infinity. We will formulate the upcoming theorem in the simplest case that for each $u_{0}$ there is a single nonzero steady-state solution. This theorem can be modified for the more general case in a straightforward manner. We, however, choose not to do so in order to keep the proof reasonably short.

TheOrem 3. Let $u(t)$ be a periodic piecewise continuous function of period 1 and consider as in (21) the functions $u^{\omega}$ of period $1 / \omega$ and their corresponding periodic solutions $B^{\omega}, X_{u}^{\omega}, X_{u d}^{\omega}, X_{a}^{\omega}, X_{a d}^{\omega}, v^{\omega}, L^{\omega}$ of the system (5)-(11). Assume that for each $u_{0} \in\{u(t) ; t \in \mathbb{R}\}$ there is a unique nonzero continuous steady-state solution with boundary condition $B(L)=u_{0}$. Let $J\left(u_{0}\right)$ and $J_{L}\left(u_{0}\right)$ be the values of the two functionals (14)-(15) for this steady-state solution. Then

$$
\begin{aligned}
& \lim _{\omega \rightarrow 0+} J^{\omega}=\lim _{\omega \rightarrow 0+} \omega \int_{0}^{1 / \omega} \int_{0}^{L^{\omega}(t)}\left(X_{a}^{\omega}(x, t)+X_{u}^{\omega}(x, t)\right) d x d t=\int_{0}^{1} J(u(t)) d t, \\
& \lim _{\omega \rightarrow 0+} J_{L}^{\omega}=\lim _{\omega \rightarrow 0+} \omega \int_{0}^{1 / \omega} L^{\omega}(t) d t=\int_{0}^{1} J_{L}(u(t)) d t .
\end{aligned}
$$

Remark. In the special case of on-off dosing with function $u(t)=u_{0} / \mu$ on $[0, \mu]$ and $u(t)=0$ on $(\mu, 1]$ these two formulas simplify to

$$
\lim _{\omega \rightarrow 0+} J^{\omega}=\mu J\left(u_{0} / \mu\right)+(1-\mu) \frac{\alpha}{\sigma}, \quad \lim _{\omega \rightarrow 0+} J_{L}^{\omega}=\mu J_{L}\left(u_{0} / \mu\right)+(1-\mu) \frac{\alpha}{\sigma} .
$$

Proof. Assume first that $u$ is continuous. Since $\omega \rightarrow 0+$ means that the period length goes to infinity and since $u$ is a periodic function, it follows that the stretched function $u^{\omega}$ is extremely slowly varying, i.e., given $\varepsilon>0$ and an arbitrary large $M>0$ one can find sufficiently small $\omega>0$ such that

$$
\left|u^{\omega}(t+\tilde{t})-u^{\omega}(t)\right|<\varepsilon \quad \text { for all } t \in \mathbb{R} \text { and }|\tilde{t}|<M .
$$

Hence, for any fixed $t$ the function $u^{\omega}$ on the large interval $[t-M, t+M]$ is almost constant and approximately equal to $u^{\omega}(t)$. What this means is that, regardless of the values of $X_{u}^{\omega}, X_{a}^{\omega}, L^{\omega}$ at time $t-M$, as time progresses to $t$ these functions will be closer and closer to the values of functions for the steady-state solution with 
boundary value $u^{\omega}(t)$. Recall that we assume there is a unique stable nonzero steadystate solution with this boundary condition. Therefore, the same will be true about the values of functionals $J$ and $J_{L}$, i.e., for some small $\delta>0$ at time $t$ :

$$
\begin{aligned}
\left|\int_{0}^{L^{\omega}(t)}\left(X_{a}^{\omega}(x, t)+X_{u}^{\omega}(x, t)\right) d x-J\left(u^{\omega}(t)\right)\right| & <\delta, \\
\left|L^{\omega}(t)-J_{L}\left(u^{\omega}(t)\right)\right| & <\delta .
\end{aligned}
$$

Here $\delta$ depends on $\omega$ and $\delta \rightarrow 0+$ as $\omega \rightarrow 0+$. Once we have this estimate, we integrate over the time interval $[0,1 / \omega]$ and average (divide by $1 / \omega$ ) to obtain

$$
\begin{array}{r}
\left|\omega \int_{0}^{1 / \omega} \int_{0}^{L^{\omega}(t)}\left(X_{a}^{\omega}(x, t)+X_{u}^{\omega}(x, t)\right) d x d t-\omega \int_{0}^{1 / \omega} J\left(u^{\omega}(t)\right) d t\right|<\delta, \\
\left|\omega \int_{0}^{1 / \omega} L^{\omega}(t) d t-\omega \int_{0}^{1 / \omega} J_{L}\left(u^{\omega}(t)\right) d t\right|<\delta .
\end{array}
$$

Now, we realize that $\omega \int_{0}^{1 / \omega} J\left(u^{\omega}(t)\right) d t=\int_{0}^{1} J(u(t)) d t$ and $\omega \int_{0}^{1 / \omega} J_{L}\left(u^{\omega}(t)\right) d t=$ $\int_{0}^{1} J_{L}(u(t)) d t$, and hence the conclusion (46) holds.

If $u$ is piecewise continuous, the only modification comes at the jump points of functions $u$. Here briefly for some fixed time period $T_{0}$ the value of functionals might differ significantly from the steady-state solution. However, as the total period $T$ goes to infinity, the ratio $T_{0} / T \rightarrow 0$; hence in the long run this jump does not matter.

It follows from Theorem 3 that, in the limit $\omega \rightarrow 0$, it is the concavity/convexity of the function $J(u)$ that determines whether or not periodic dosing is better than constant dosing.

Theorem 4. Let $J$ be the function defined in Theorem 3; that is, $J(u)$ is the value of the functional (14) for the steady-state solution with constant dose u. Assume that $J$ is a concave down function on the interval $\left[A_{1}, A_{2}\right]$ and $u(t) \in\left[A_{1}, A_{2}\right]$. Then

$$
\lim _{\omega \rightarrow 0_{+}} J_{\omega}=\int_{0}^{1} J(u) d t<J\left(u_{0}\right) ;
$$

i.e., for sufficiently small frequencies $\omega$, periodic dosing is more effective than constant dosing with dose $u_{0}$. Conversely, if $J$ is concave up on the whole interval $\left[A_{1}, A_{2}\right]$ and $u(t) \in\left[A_{1}, A_{2}\right]$, then for sufficiently small frequencies $\omega$, periodic dosing is sure to be less effective than constant dosing with dose $u_{0}$. Similar claims also hold for the functional $J_{L}$.

Proof. We expand $J$ around $u_{0}$ using its Taylor formula, i.e.,

$$
J(u)=J\left(u_{0}\right)+J^{\prime}\left(u_{0}\right)\left(u-u_{0}\right)+\frac{J^{\prime \prime}\left(\xi_{u}\right)}{2}\left(u-u_{0}\right)^{2},
$$

where $\xi_{u}$ is some number in the interval between $u$ and $u_{0}$. Next, we replace $u$ by $u(t)$ and integrate $J$ over interval $[0,1]$.

$$
\lim _{\omega \rightarrow 0_{+}} J_{\omega}=\int_{0}^{1} J(u(t)) d t=J\left(u_{0}\right)+\int_{0}^{1} \frac{J^{\prime \prime}\left(\xi_{u}(t)\right)}{2}\left(u(t)-u_{0}\right)^{2} d t .
$$

The claim then follows; if $J^{\prime \prime}<0$, the right-hand side is smaller than $J\left(u_{0}\right)$ for small values of $\omega$. On the other hand, if $J^{\prime \prime}>0$, the opposite is true. 


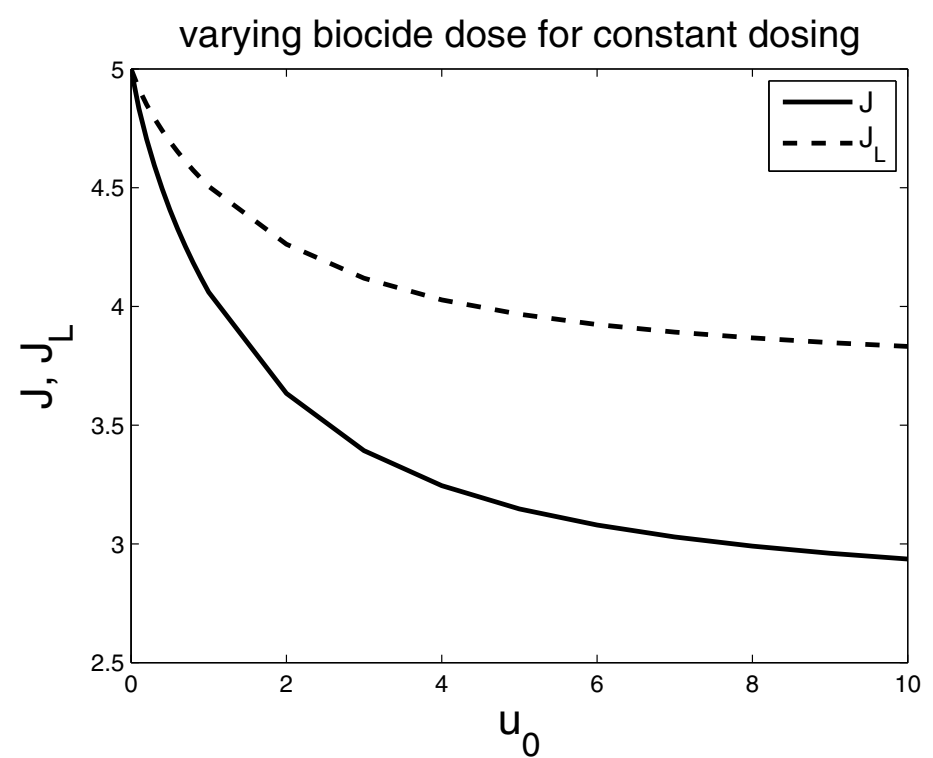

(a)

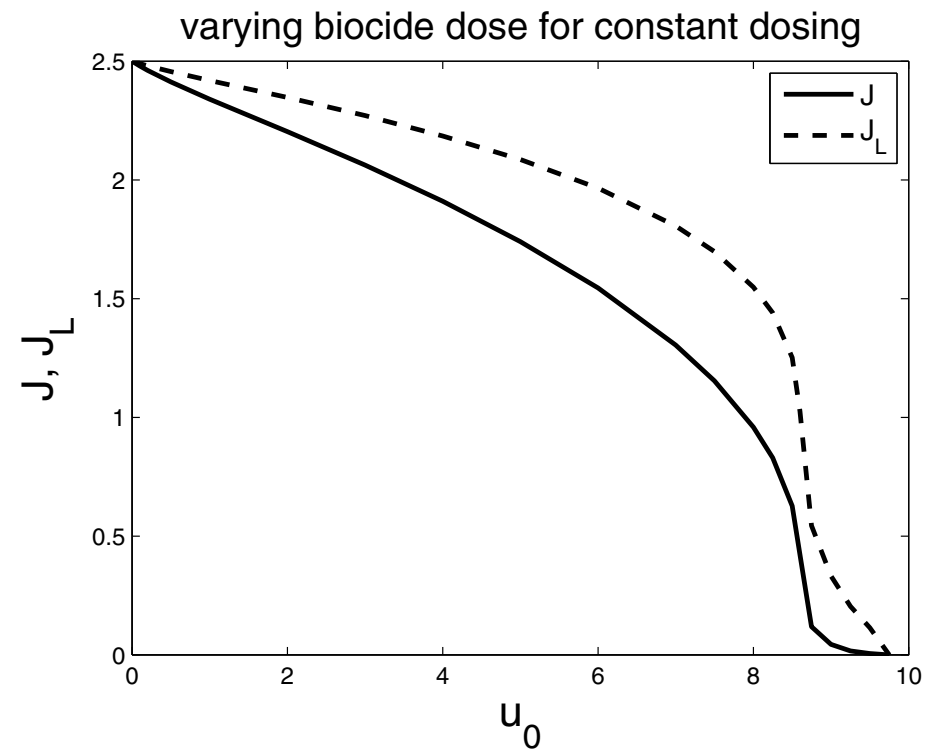

(b)

FIG. 1. (a) Concave-up functionals $J$ and $J_{L}$ for different values of the biocide dose $u_{0}$ under constant dosing. The values of the parameters are $L(0)=5, \phi=1, \lambda=1, \alpha=1, \gamma=0.1, \sigma=0.2$, $M_{\psi}=0.2, k=0.1, M_{\phi}=2$. (b) Concave-down functionals $J$ and $J_{L}$ for different values of the biocide dose $u_{0}$ under constant dosing. The values of the parameters are $L(0)=2.5, \phi=1, \lambda=0.1$, $\alpha=1, \gamma=0.01, \sigma=0.4$, and the functions $\phi, \psi$ are chosen to be $\phi(B)=B, \psi(B)=B$.

Example of functionals $J, J_{L}$ that are concave up for all $u$ are shown in Figure $1(\mathrm{a})$, and those that are concave down over an interval $(u \in[0,8.5))$ are shown in Figure 1(b). In the latter case, for sufficiently small frequencies, periodic dosing 
will be more effective than constant dosing only for $u_{0}<8.5$. We note here that asymptotic expansions (up to second order) of the functionals $J, J_{L}$ about $u_{0}=0$ and $u_{0}=\infty$ were done in [38] for the cases $M_{\phi}=\infty, M_{\psi}=\infty$ and $M_{\phi}=\infty, M_{\psi}=0$, respectively. A notable result for thin biofilms $\left(\phi \alpha \sigma^{-1} \ll 1\right)$ is that for sufficiently small and large values of $u_{0}$, the functionals $J, J_{L}$ are concave up. For the new scenar$\operatorname{ios} M_{\phi}<\infty, M_{\psi}<\infty$ presented here, a similar statement about thin biofilms holds only for small values of $u_{0}$. Dose-response curves in in vitro studies of planktonic cells under constant dosing regiment for different doses of antibiotics might prove helpful in validating our result (e.g., see Figure 2 in [31]). However, more data are necessary to make clear statements about the dose-response convexity for small doses of antimicrobials. For thick biofilms $\left(\phi \alpha \sigma^{-1} \gg 1\right)$, both concave up and down response curves are possible (see Figure 1). This ambiguity in convexity for small doses of antibiotics for biofilm cells apparently can be observed in a number of studies (for example, Figure 1 in [1] shows possibly convex up; Figures 1-2 in [8] show possibly convex down). Thus convexity characterization is quite possibly antimicrobial and/or biofilm dependent.

As a consequence of Theorem 4, we have the following corollary.

Corollary 1. Let $J(u)$ be the value of the functional (14) for the steady-state solution with constant dose $u$. If for all $u$ we have $J(u)>0$, then $J$ is a concave-up function for sufficiently large values of u; hence periodic dosing for large doses and small frequencies is less effective than constant dosing. Similar claims also hold for the functional $J_{L}$.

Remark. $J^{\prime \prime}<0$ (or $J_{L}^{\prime \prime}<0$ ) signifies that an increase in dosage is surprisingly effective in the sense that the acceleration in decrease of $J$ (or $J_{L}$ ) is greater than the acceleration in increase of $u$, for example, because antimicrobial penetration suffers from diffusive-reactive barriers. In this case it is best to vary dosage in time so as, at least for short times, to expose the biofilm to the particularly effective higher dosages even at the expense of using lower dosages at other times. In the reverse case $J^{\prime \prime}>0$ (or $J_{L}^{\prime \prime}>0$ ), the opposite is true: relative ineffectiveness of higher dosing (due, for example, to antimicrobial saturation) does not justify use of variable dosing. According to Corollary 1, for recalcitrant biofilms such that $J(u)>0$ for all $u$, constant application is optimal for large enough dosage.

4. Simulated dosing experiments. Section 4.1 describes numerical methods used in simulations. Section 4.2 presents two important assertions concerning onoff dosing (Corollaries 2-3), and, finally, section 4.3 presents an example application dosing experiment where the effects of one concentrated dose and two half-doses are compared.

4.1. Numerical details. So far, parameter choices have been arbitrary up to some mild requirements. For numerical purposes we now specify the functions $\varphi, \psi$ to be $\varphi(B)=\frac{M_{\varphi} B}{B+k}, \psi(B)=\frac{M_{\psi} B}{B+k}$ (standard saturation forms). We note here that if the growth rates $\alpha_{u}$ and $\alpha_{a}$ for the unadapted and adapted cells differ, for $u_{0}=0$ the biofilm thickness $L$ is only controlled by $\alpha_{u}$. On the other hand, for significant doses $u_{0}$, the biofilm thickness $L$ in most cases is only controlled by $\alpha_{a}$. We will thus continue to set $\alpha_{u}=\alpha_{a}$. In actuality, $\alpha_{u}$ is probably slightly larger than $\alpha_{a}$ as adaptation mechanisms come with some cost, but a small difference is not of much effect here.

We make estimates as follows for the ratios $\alpha / \gamma, \lambda / \gamma$, and $\alpha / \sigma$ in case of adaptation based on experimental observations [33]. It is estimated that the reversion rate $\gamma$ is 0.01 to 1 times the growth rate $\alpha$ consistent with observations; see, e.g., 
$[19,5]$. However, in the case when the adapted cells are not killed by the biocide $\left(M_{\psi}=0\right)$, the only relevant situation for our model is $\alpha / \gamma=o(1)$. Otherwise, if $\alpha \gg \gamma$ and $M_{\psi}=0$, the biocide would have negligible effect on the biofilm thickness, that is to say, it would hardly function as a biocide at all and thus not be of practical interest. Indeed, it can be shown that for $u_{0}=0$ the steady-state solutions satisfy $X_{u}+X_{a}=1$ and $L=\frac{\alpha}{\sigma}$, while when $u_{0} \rightarrow \infty$, it can be shown that $X_{u}+X_{a} \geq 1-\frac{\gamma}{\alpha}$ and $L \geq \frac{\alpha-\gamma}{\sigma}$, i.e., the biofilm thickness almost does not change compared to no dosing if $\gamma / \alpha$ is much less than 1 . The ratio $\lambda / \gamma$ of reversion to disinfection rates has influence on the relative population sizes of the viable cells. For small biocide doses it is easy to show that $X_{u} \approx \frac{\gamma}{\lambda+\gamma}$ and $X_{a} \approx \frac{\lambda}{\lambda+\gamma}$, i.e., $X_{a} / X_{u} \approx \lambda / \gamma$. Disinfection of unadapted cells may typically require about $1-2$ hours, whereas a reasonable time for reversion of adapted cells seems to be about 10 hours. Hence, the ratio $\lambda / \gamma$ is set in the range $0.1-0.2$. Finally, we take the detachment coefficient to be $0.1-0.2$ times the growth rate $\alpha$.

The simulations to follow use a second order explicit Runge-Kutta method. Our approach is motivated by techniques used to prove the existence and uniqueness of solutions of the PDE system in [37] and hence is based on the method of characteristics. Equation (1) can be rewritten as

$$
\frac{\partial X_{u}}{\partial t}+\frac{\partial X_{u}}{\partial x} v=-X_{u} \frac{\partial v}{\partial x}-(\varphi(B)+\lambda(B)) X_{u}+\alpha X_{u}+\gamma X_{a} .
$$

The left-hand side in (51) is the directional derivative of $X_{u}$ in the $(x, t)$ plane in the direction $(v, 1)$. The flow of the vector field $(v, 1)$ defines characteristics with characteristic equations

$$
\frac{d x}{d t}=v(x(t), t), \quad x(0)=x_{0} .
$$

The time-axis $x=0$ is also a characteristic, since $v(0, t)=0$. Hence, the left-boundary conditions for the solutions can be obtained from the method directly. We observe that with passing time the distance between characteristics will increase. If we let this happen for a sufficiently long time, we will lose precision since distances between points on the moving grid will increase. To avoid this, we introduce a new characteristic at the moment when the distance between the original characteristics is greater than $2 d$, where $d$ is the initial spatial step-size. We compute the initial values on the new characteristic using second order interpolation.

All quantities are computed in two stages, first by computing the preliminary values using Euler's method and then by computing the "real values" by approximating the average slope using the preliminary values. Once the values of $X_{u}, X_{a}$ are obtained as described before, $v(i, j)$ is calculated from (11) with a trapezoid method.

Since we have a moving boundary, the velocity at the interface at time $i$ is computed as the weighted average value of the velocities $v(i, j-1)$ and $v(i, j)$ for some appropriate index $j$ such that $x(i, j-1)$ is the closest point on the characteristic at time $i$ to the left of $L(i)$, and $x(i, j)$ is on a characteristic to the right of the interface $L(i)$. Note that the equation for $L$ is $\dot{L}=v(L,)-.\sigma L^{2}$. Hence, in the case $\sigma=0$ (no detachment) $L$ would just be a characteristic. When $\sigma>0, L(i)$ is always to the left of the last grid point $x(i, j)$, so $L(i)$ is computed via interpolation (no extrapolation is required).

4.2. Comparing simulation and analytical results. As already mentioned in section 2, if $u$ is periodic, then solutions of the system (5)-(11) become periodic 


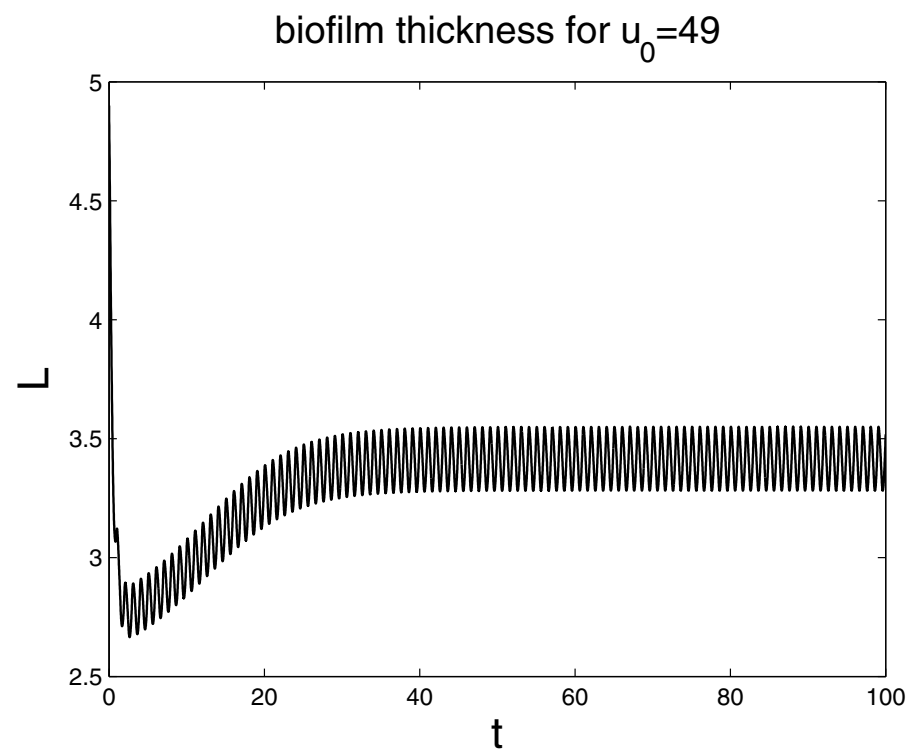

(a)
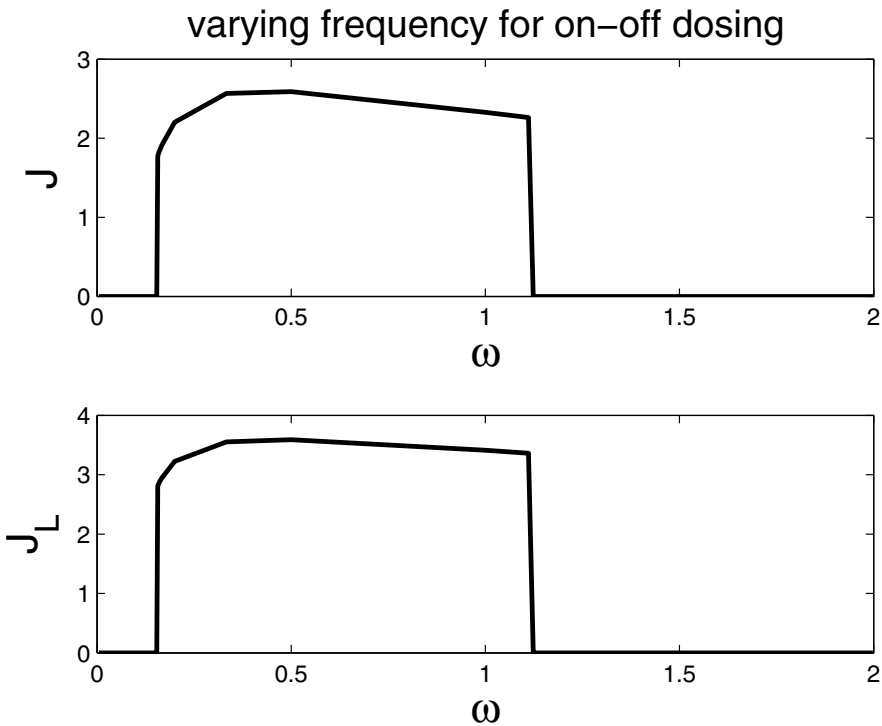

(b)

FIG. 2. (a) Biofilm thickness $L$ with on-off dosing for values of the parameters $L(0)=4.9$, $u_{0}=49, \phi=1, \lambda=2, \alpha=2, \gamma=0.2, \sigma=0.4, M_{\psi}=10, k=100, M_{\phi}=100, \mu=0.5, P=1$. (b) Functionals $J$ and $J_{L}$ for different values of the frequency $\omega$. The values of the other parameters are the same as in (a).

as $t \rightarrow \infty$ with the same period as the function $u$. In some cases, depending on the parameters, the limiting solution can be nonzero; in other cases, only the trivial limit occurs. This is demonstrated in Figures $2(\mathrm{a})$ and $3(\mathrm{a})$, where the biofilm thickness $L$ is shown for on-off dosing $\left(u(t)=u_{0} / \mu\right.$ on $[0, \mu)$ and $u(t)=0$ on $\left.[\mu, 1)\right)$ with frequency 


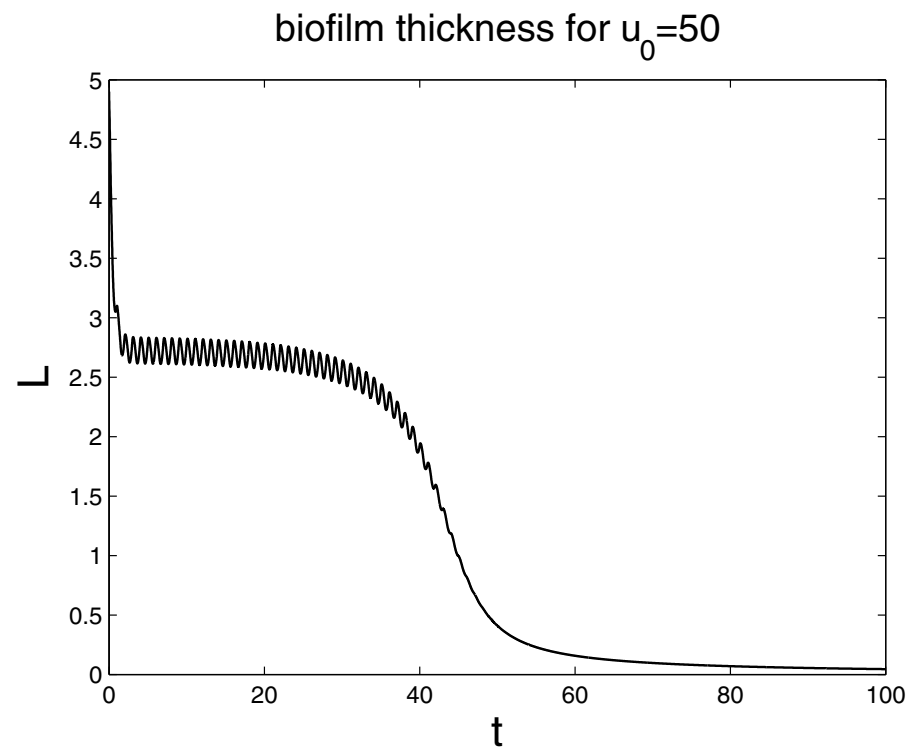

(a)

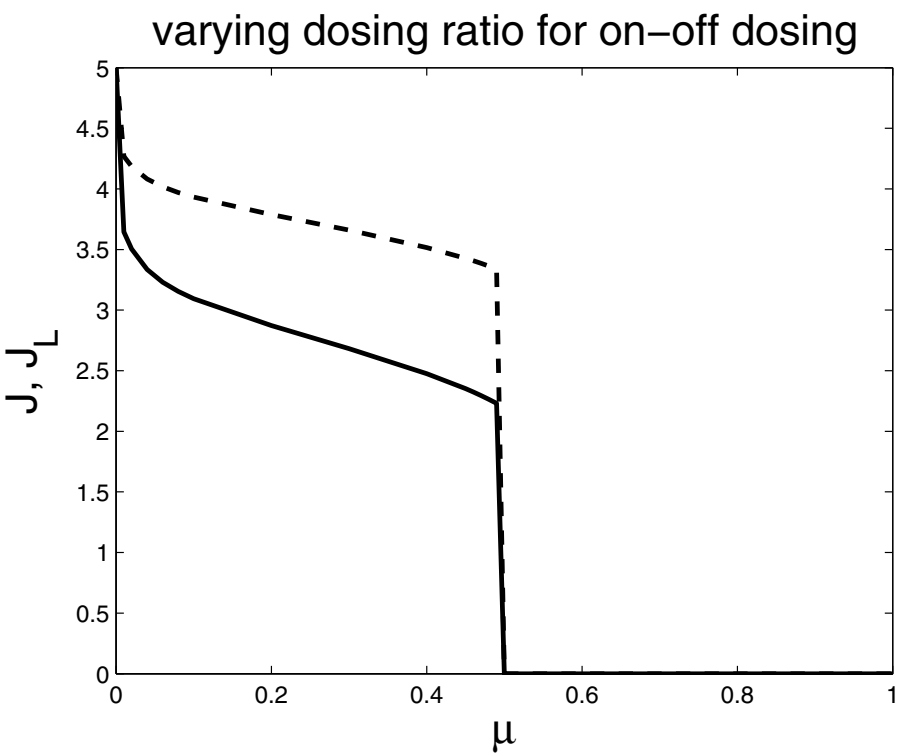

(b)

FIG. 3. (a) Biofilm thickness $L$ with on-off dosing for values of the parameters $L(0)=4.9$, $u_{0}=50, \phi=1, \lambda=2, \alpha=2, \gamma=0.2, \sigma=0.4, M_{\psi}=10, k=100, M_{\phi}=100, \mu=0.5, P=1$. (b) Functionals $J$ and $J_{L}$ for different dosing time/period $\mu$. The values of the other parameters are the same as in (a).

$\omega=1$ and average biocide dose $u_{0}=49$ and $u_{0}=50$, respectively. There exists a bifurcation point between the values of 49 and 50, where the long-term behavior of the solution suddenly changes; a slight increase in the dose leads to eradication of the biofilm. The same effect can also be achieved by slightly decreasing the frequency, but 
maintaining the average dose (see Figure 2(b)). Notice that when the frequency of dosing goes to zero or infinity the biofilm is eradicated, while in between the long-term limit is nonvanishing. Eradication in the case $\omega \rightarrow \infty$ directly follows from Theorem 2 since the steady-state solution is trivial, though for the case $\omega \rightarrow 0+$ we only have numerical evidence but note that small $\omega$ means long intervals of nearly full dosing.

As we mentioned in the introduction, if both functions $\varphi, \psi$ are chosen to be linear and we consider an on-off dosing $u^{\mu}(t)=u_{0} / \mu$ on $[0, \mu]$ and $u^{\mu}(t)=0$ on $(\mu, 1]$ for a parameter $\mu \in(0,1]$, then we obtain an unrealistic prediction for optimal dosing strategy. As follows from Theorem 2, for large $\omega$ (i.e., small period $P$ ) the term $\mu \lambda$ in (23) and (25) goes to zero as the parameter $\mu \rightarrow 0+$ with the consequences that the functionals $J(\mu)$ and $J\left(\mu_{L}\right)$ attain their minima at $\mu=0$. It follows that the optimal dosing strategy is a delta function ( $\operatorname{since} \lim _{\mu \rightarrow 0} u^{\mu}=u_{0} \delta$ ). One would instead expect saturation at a sufficiently high dosage.

This can be corrected by choosing the functions $\varphi, \psi$ to be bounded, as in Figure 3 (b). In this case we can actually prove that the minimum is attained for a positive value of $\mu \leq 1$ (see Corollary 2 below). This indeed happens in Figure 3(b) (where we use the same parameters as in Figure 3(a) but vary $\mu$, the dosing ratio). Here the minimum of the functionals $J, J_{L}$ is attained at all values $\mu \geq \mu_{0}>0$ for a threshold value $\mu_{0} \approx 1 / 2$.

Corollary 2. Let $u_{0}>0$. Assume that the functions $\varphi$ and $\psi$ are sublinear, i.e.,

$$
\lim _{x \rightarrow \infty} \frac{\varphi(x)}{x}=\lim _{x \rightarrow \infty} \frac{\psi(x)}{x}=0 .
$$

Consider the functionals $J(\mu)$ and $J_{L}(\mu)$ for on-off dosing with the same average dose $u_{0}$ and for dosing time $\mu$. Then the minimum of $J$ and $J_{L}$ is attained at some $\mu>0$.

Proof. Let us analyze what happens in (5)-(11) as $\mu \rightarrow 0+$. During the on time, $\varphi\left(B^{\mu}(t)\right)>0, \psi\left(B^{\mu}(t)\right)>0$, and during the off time, $\varphi\left(B^{\mu}(t)\right)=\psi\left(B^{\mu}(t)\right)=0$. The assumption of sublinearity implies

$$
\int_{0}^{P} \varphi\left(B^{\mu}\right) d t=\int_{0}^{\mu} \varphi\left(\frac{u_{0}}{\mu} \frac{\cosh (\phi x)}{\cosh \left(\phi L^{\mu}(t)\right)}\right) d t \leq \mu \varphi\left(\frac{u_{0}}{\mu}\right),
$$

so that $\lim _{\mu \rightarrow 0+} \varphi\left(B^{\mu}\right)=\lim _{\mu \rightarrow 0+} \psi\left(B^{\mu}\right)=0$ in $L^{1}(0, P)$. Hence, the contributions of these two terms in (5)-(11) go to zero as $\mu \rightarrow 0+$. We can conclude that as $\mu \rightarrow 0+$, the functionals $J(\mu)$ and $J_{L}(\mu)$ converge to the no-dosing situation, i.e.,

$$
\lim _{\mu \rightarrow 0} J(\mu)=\lim _{\mu \rightarrow 0} J_{L}(\mu)=\frac{\alpha}{\sigma} .
$$

This means the minimum will never be attained at $\mu=0$.

Remark. For comparison, if, for example, $\varphi$ is linear, then $\varphi\left(B^{\mu}(t)\right) \rightarrow c \sum_{n \in \mathbb{Z}} \delta(n P)$, and hence the contribution of this term in the limit $\mu \rightarrow 0+$ cannot be ignored.

Figure $4(\mathrm{a})$ plots the functionals $J$ and $J_{L}$ versus the frequency $\omega$ (on $\log$ scale) for on-off dosing. The two horizontal lines represent the limits $\omega \rightarrow 0+$ and $\omega \rightarrow \infty$ computed explicitly by invoking Theorems 2 and 3 .

Unlike Figure $3(\mathrm{~b})$, where functionals $J, J_{L}$ attain minimum at $\mu=1$ (i.e., constant dosing), Figure 4(b) shows a situation when on-off dosing is more advantageous compared to constant dosing with an optimal value of $\mu$ strictly between 0 and 1 . The gain of the functionals $J, J_{L}$ over constant dosing is about $20 \%$ and $10 \%$, respectively. 

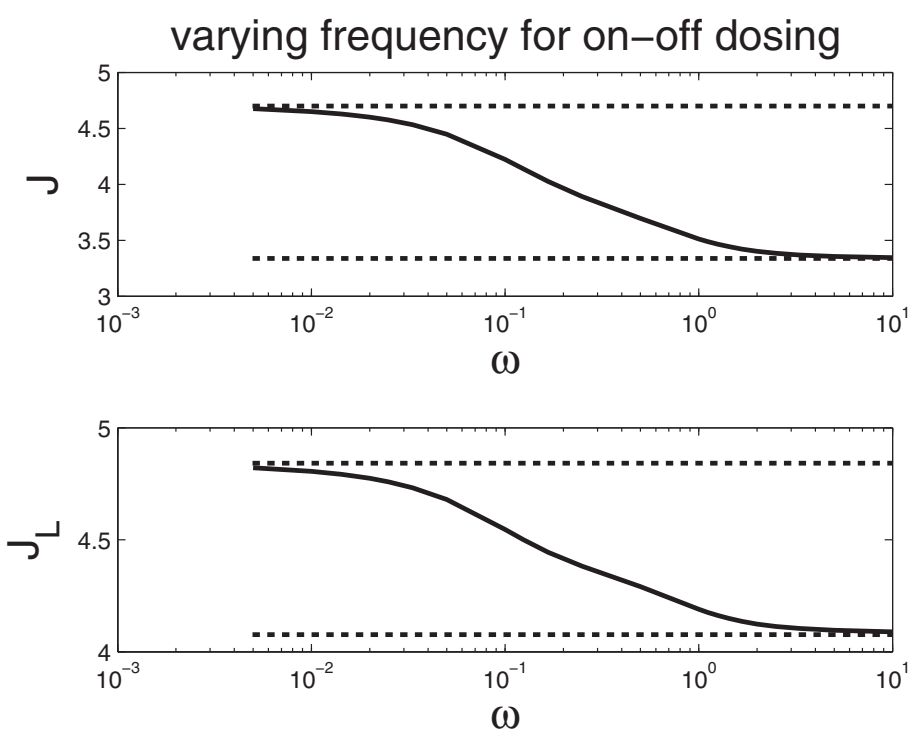

(a)

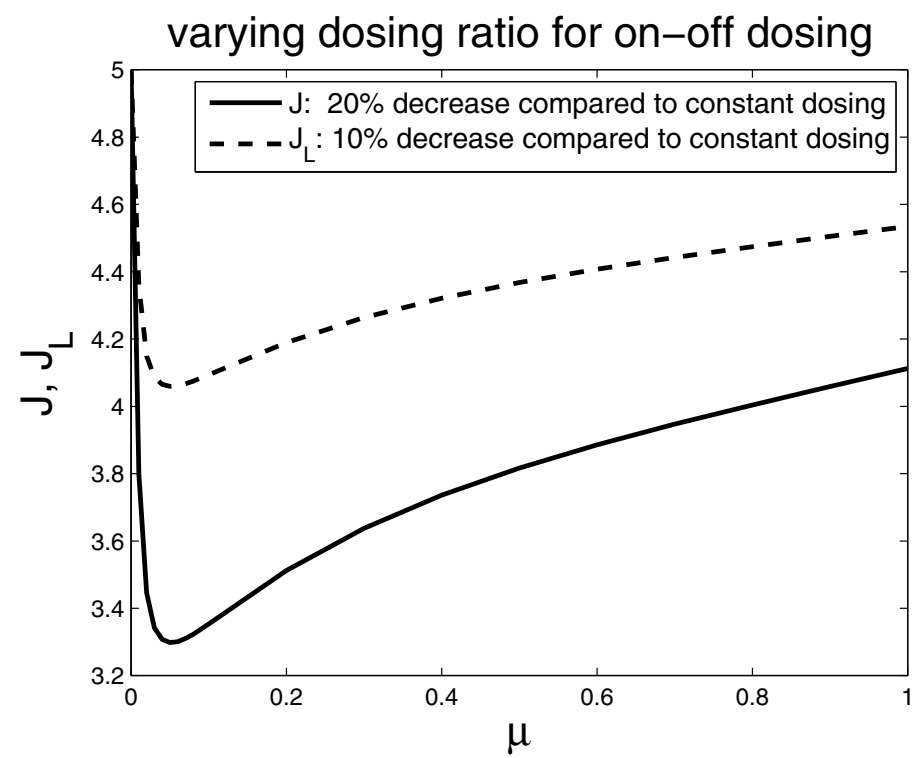

(b)

FIG. 4. (a) Functionals $J$ and $J_{L}$ for different values of the frequency $\omega$. The values of the parameters are $L(0)=1, u_{0}=1, \phi=1, \lambda=2, \alpha=2, \gamma=0.4, \sigma=0.4, M_{\psi}=0, k=1, M_{\phi}=35$, $\mu=0.2$. The dotted horizontal lines, demarcating $\omega \rightarrow 0$ and $\omega \rightarrow \infty$, were computed explicitly based on Theorems 2 and 3. (b) Functionals $J$ and $J_{L}$ for different ratios of the dosing time/period. The values of the parameters are $L(0)=1, u_{0}=1, \phi=1, \lambda=2, \alpha=2, \gamma=0.4, \sigma=0.4, M_{\psi}=0$, $k=1, M_{\phi}=35, P=1$.

Figure 5 compares constant dosing to on-off dosing with the dosing ratio $\mu=0.05$. The average dose $u_{0}$ is varied. Notice that for small average dose, constant dosing is always worse than on-off dosing. This is due to the fact that for small $u_{0}$ (and 

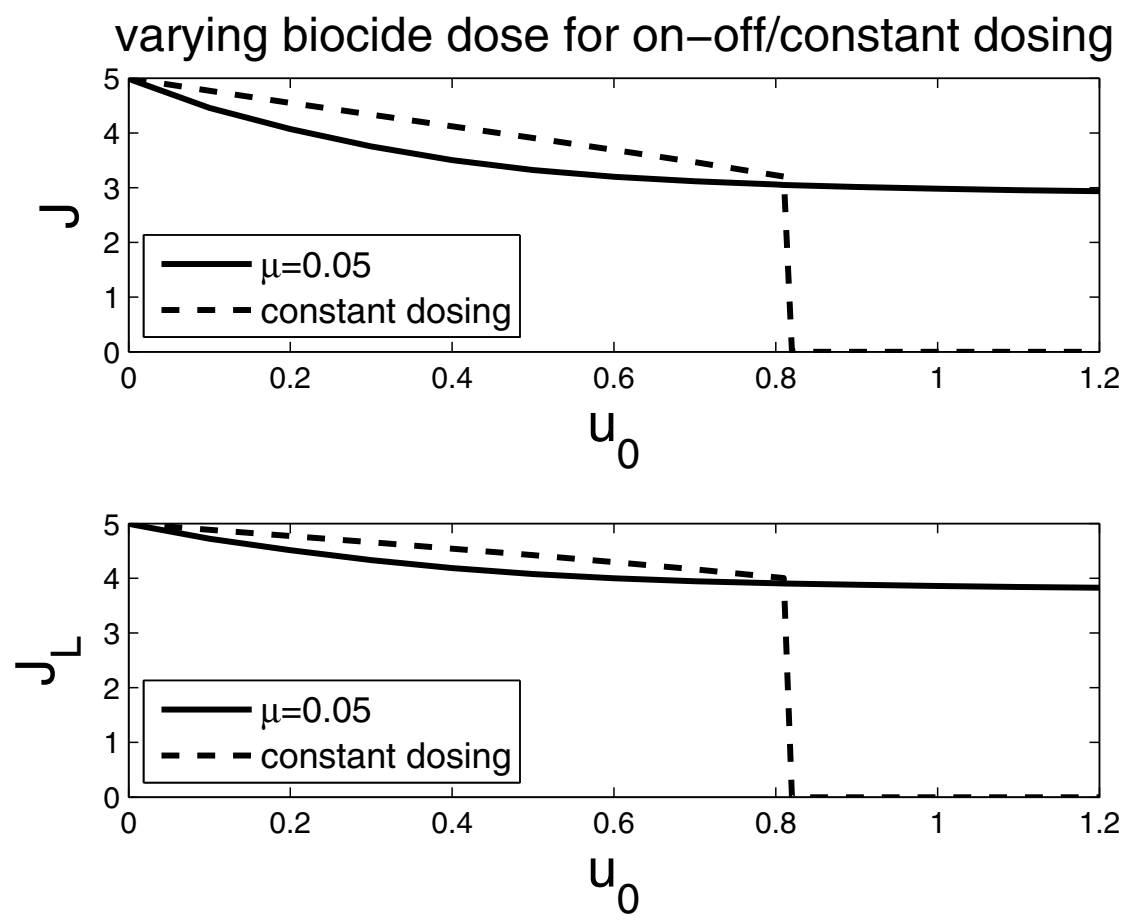

Fig. 5. Functionals $J$ and $J_{L}$ for different values of the biocide dose $u_{0}$. The values of the parameters are $L(0)=1, \phi=1, \lambda=2, \alpha=2, \gamma=0.4, \sigma=0.4, M_{\psi}=3.5, k=1, M_{\phi}=35$. The full curve corresponds to constant dosing with the same parameters. The dashed curve corresponds to on-off dosing with $\mu=0.05, P=1$.

hence small $B$ ) the term $\varphi(B)$ is almost linear and the effect of saturation of the bounded function $\varphi$ is negligible (see Corollary 3). The situation changes if $u_{0}$ is large; for this set of parameters the best strategy is constant dosing. Indeed, recall that $\varphi(B)=\frac{M_{\varphi} u_{0}}{u_{0}+\mu k}$ for $B=\frac{u_{0}}{\mu}$ and $\mu \in(0,1]$. Since $\mu k \approx 0$ and $M_{\varphi} \gg 1$, we obtain that $\varphi(B) \approx M_{\varphi}$ during the dosing time. The largest dosing time is when $\mu=1$ (whole time), and hence $\mu=1$ is best; spiking dosage by setting $\mu<1$ is of no advantage in the saturated case.

Corollary 3. Let $\mu>0$. Then for all $u_{0}>0$ sufficiently small, on-off dosing with average dose $u_{0}$ and dosing ratio $\mu$ is better than constant dosing with dose $u_{0}$.

Proof. As stated above, if $u_{0}$ is small, then $\varphi(B) \approx a B$ and $\psi(B) \approx a^{\prime} B$ for some $a>0$ and $a^{\prime}>0$. It follows that the functions $\Phi$ and $\Psi$ appearing in the statement of Theorem 2 will be approximately equal to $a B$ and $a^{\prime} B$, respectively. Consider the linear case of the system (5)-(11), where the terms $\varphi(B), \psi(B)$ are replaced by $a B$ and $a^{\prime} B$. The first order expansion of the functional $J_{L}$ about $u_{0}=0$ yields $J_{L}=L^{(0)}+L^{(1)} u_{0}+O\left(u_{0}^{2}\right)$, where $L^{(0)}=\frac{\alpha}{\sigma}$ and

$$
L^{(1)}=-\frac{a \gamma /(\lambda+\gamma)+a^{\prime} \lambda /(\lambda+\gamma)}{\alpha \phi \cosh \left(\phi L^{(0)}\right)} \int_{0}^{L^{(0)}} \frac{\sinh (\phi x)}{x} d x<0 .
$$

(The analysis is similar for the functional $J$.) From this the claim follows.

Remark. Note that "sufficiently small" dosage means that $\varphi, \psi$, are within their linear regimes. 
4.3. A simple computational dosing experiment. As an example application, we consider the following question: Given an adaptive infection, what is the preferable dosing strategy? That is, how should a daily dosage be divided into pills per day? From practical considerations, a patient might rather take one large pill than a number of smaller ones. However, disinfection efficacy may or may not be well served by convenience, although at least some evidence supports one per day dosing $[19,5,6]$. We won't present an exhaustive study of model predictions for this question here, but for example purposes we will discuss a comparison of a regime consisting of one full-dosed pill per day versus a regime of two half-dosed pills per day.

Figure 6 shows biofilm thickness $L$ versus time for different values of the Thiele modulus $\phi$ (Thiele modulus larger than 1 indicates biofilm thickness large enough to prevent full penetration; Thiele modulus less than 1 indicates full penetration is possible). We tested two scenarios: a concentrated dose at the beginning of the dosing period and two half-dosages given at the beginning and the middle of the time period, both delivering antimicrobial for the same length of dosing time as the concentrated dose. We observe that for thinner biofilms $(\phi=0.8, \phi=1)$ a single concentrated dose is better than two half-doses. Indeed, the functional $J_{L}$ for the single dose corresponding to the $\phi$ 's is about $28 \%$ and $23 \%$ smaller compared to the functional $J_{L}$ for double dose. For thick biofilms $(\phi=2)$, however, the double dosing was slightly more advantageous compared to the single dose, although differences were small and neither were very effective.

Figure 7 shows biofilm thickness $L$ versus time for different values of the growth rate $\alpha$. Recall that for no-dosing the steady state is $L=\alpha / \sigma$. We conclude a similar phenomenon as before: For thinner biofilms $(\alpha=0.5, \alpha=1)$ a single dose is better than double dosing with differences $12 \%$ and $33 \%$ corresponding to the $\alpha$ 's. These results indicate that the success of dosing strategies is tied to biofilm thickness and, under the assumptions made here, a small concentrated dosage is often more effective than multiple dosing. As a remark, the thin biofilm case (where a single concentrated pill is particularly effective) is rather similar to a planktonic infection in that "thin" means that biocide effectively penetrates, reaching all organisms. Thick biofilms, at least according to our model, are effectively defended against disinfection by adaptive response, pointing towards the importance of timeliness against bacterial infections. Indeed, mature biofilms are well known to exhibit significant recalcitrance against antimicrobials.

5. Summary. In Theorem 1 we presented sufficient conditions for the existence of trivial/nontrivial steady states. In Theorems 2 and 3 the limiting behavior of periodic solutions was studied for large and small periods, which allows us to directly compare these two dosing strategies with constant dosing. For example, it was shown that for small doses of biocide, on-off dosing is always more effective than constant dosing and hence should be preferred (see Corollary 3). Numerical simulations indicated that for large doses this might not be true, and constant dosing may be preferable, depending on the death rate due to antimicrobial action. For example, in the case when the death rate is linear (in biocide), a consequence of Theorem 2 is that on-off dosing for small periods is always better than constant dosing regardless of the biocide dose. On the other hand, as a consequence of Theorem 3 we are able to state sufficient conditions under which the periodic dosing is better or worse than constant dosing. This statement was formulated in Theorem 4. To illustrate the applicability of Theorems 1-2, we performed a simple computational dosing experiment comparing the effectiveness of a single concentrated dosing versus multiple dosings (per period). 


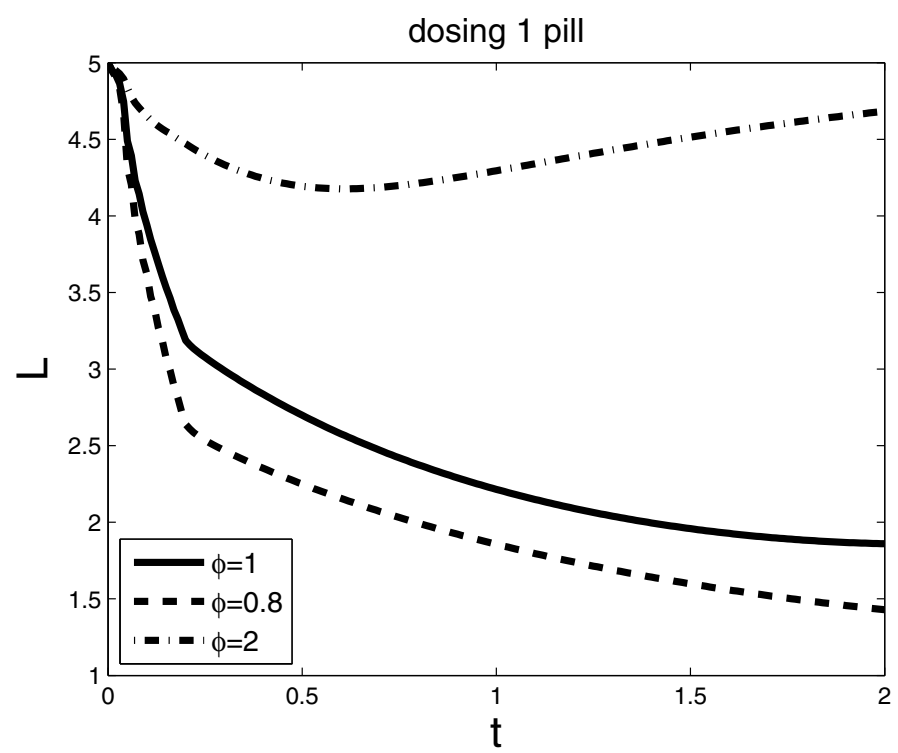

(a)

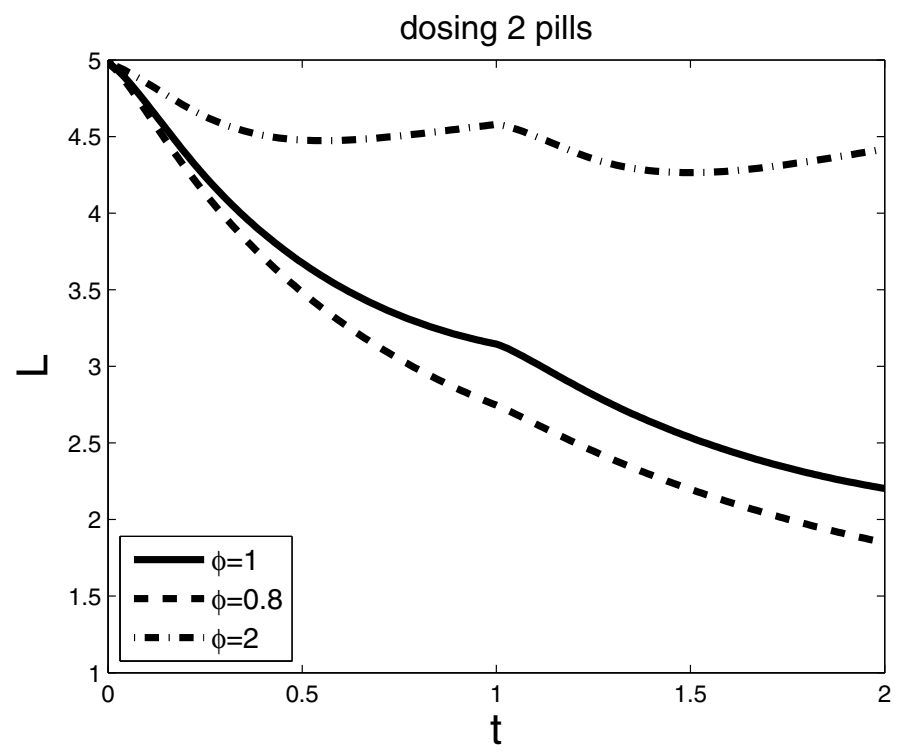

(b)

FIG. 6. (a) Biofilm thickness $L$ when one pill is given at different values of the Thiele modulus $\phi$. The values of the parameters are $L(0)=5, u_{0}=12, \lambda=2, \alpha=1, \gamma=0.2, \sigma=0.2, M_{\psi}=100$, $k=100, M_{\phi}=1000$. The time length is $T=2$ and the dosing ratio is $\mu=0.1$. (b) Biofilm thickness $L$ when two pills are given for different values of the Thiele modulus $\phi$.

Our results indicate that the thickness of a biofilm is relevant to the success of dosing: at low dosage levels (more likely to be medically relevant), thin biofilms are better treated with a single dose per period, whereas it is much harder to treat thick biofilms effectively with either a single dose or multiple doses, in agreement with general wisdom on the subject. 


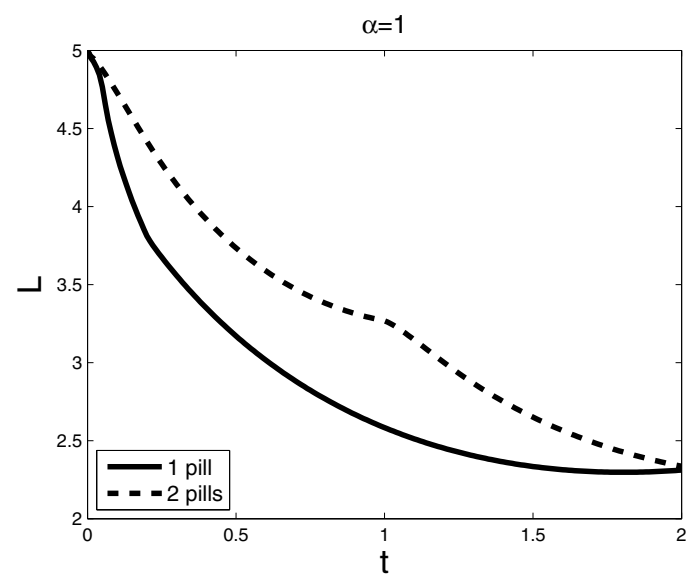

(a)

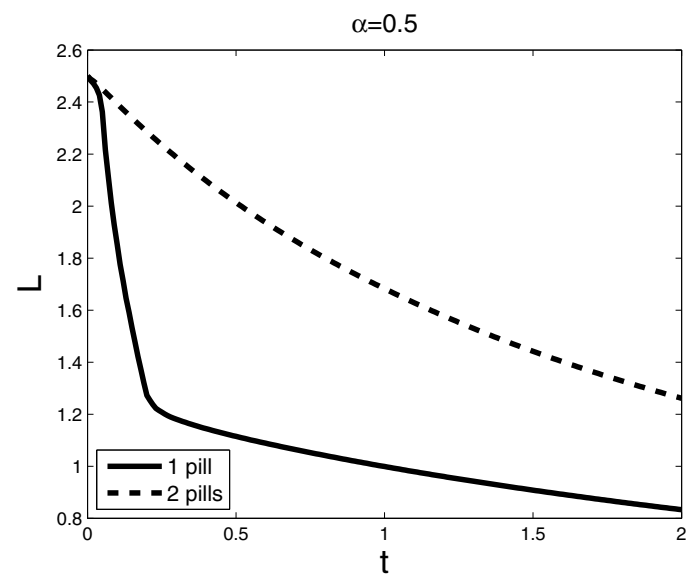

(b)

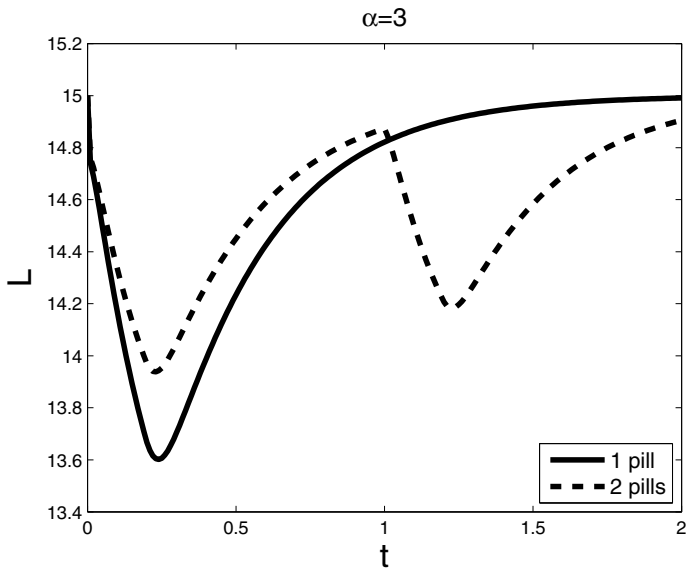

(c)

FIG. 7. Biofilm thickness $L$ when one and two pills are given for different values of the growth rate $\alpha$. The values of the parameters are $L(0)=\alpha / \sigma, u_{0}=10, \phi=1, \lambda=2, \gamma=0.2, \sigma=0.2$, $M_{\psi}=100, k=100, M_{\phi}=1000$. The time length is $T=2$ and the dosing ratio is $\mu=0.1$.

Copyright (c) by SIAM. Unauthorized reproduction of this article is prohibited. 
Although Theorems 2 and 3 deal with the asymptotic (in period length) behavior of periodic solutions, their conclusions appear to extend to relevant and reasonable regimes. For example, in Figure 4(a) the steady state is achieved for a period of length 0.5 hour and 100 hours, respectively. See also Figure 2(b), where the biofilm could be eradicated with on-off dosing for periods of length less than 1 hour and at least 5 hours. Hence, in this sense, clinical relevance is plausible. The ideal would be to corroborate our predictions with suitable experimental data. However, such data are not always available, or the measurements are not sufficiently detailed (see, e.g., the paragraph after Theorem 4), or the time scale of experiments is too short. In this case, we make testable model predictions. For example, one of our main results, namely, that on-off dosing for small doses of biocide is more effective than constant dosing, is in line with experimental observations in [25] and [13]. In [25], once-daily dosing was compared with continuous dosing in different animal models. Hence, the doses applied were within a tolerance limit ("not very large"). The efficacy of once-daily aminoglycoside doses was as great as or even greater than continuous infusion. The optimal dosing regiment, however, should also take into account drug toxicity resulting from sustained high drug levels during the time required to develop adaptive resistance (3-6 hours for $P$. aureginosa in vitro [13]) and allowing enough time for complete clearance of the drug before repeating the next cycle.

In conclusion, we have presented several results aimed towards developing mathematical techniques for understanding and designing effective dosing protocols in the face of a microbial defense mechanism. We suggest that adaptive response in wellestablished (i.e., thick) biofilms is a powerful defense. (We note in passing that while the model proposed here applies to microbial biofilms, it serves as a framework for including planktonic systems in a time-dependent model. This modification would require an additional equation for the substrate adjusted for a chemostat system and/or a batch culture.) Adaptive response in biofilms has not been a subject of much modeling to date, despite its potential impact in both medical and industrial contexts. Part of the explanation for this is the complications of the interconnection between adaptive response, a local biological phenomenon, and the spatial structure and dependence of biofilms, a coupling which introduces a number of mathematical complications. Nor has adaptive response been extensively targeted in the clinical context, again possibly as a consequence of the complicated nature of microbial infections. Nevertheless, the topic is sufficiently important that it requires attention. We hope here to have highlighted this importance and to have illustrated, at least to the extent possible through a mathematical model, the need for further clinical attention.

Acknowledgment. The authors would like to thank Phil S. Stewart for his valuable comments without which this manuscript could not have been written.

\section{REFERENCES}

[1] M. J. Ashby, J. E. Neale, S. J. Knott, and I. A. Critchley, Effect of antibiotics on nongrowing planktonic cells and biofilms of Escherichia coli, J. Antimicrob. Chemother., 33 (1994), pp. 443-152.

[2] B. P. Ayati And I. Klapper, A multiscale model of biofilm as a senescence-structured fluid, Multiscale Model. Simul., 6 (2007), pp. 347-365.

[3] N. Bagge, M. Schuster, M. Hentzer, O. Ciofu, M. Givskov, E. P. Greenberg, and N. HøIBY, Pseudomonas aeruginosa biofilms exposed to imipenem exhibit changes in global gene expression and beta-lactamase and alginate production, Antimicrob. Agents Chemother., 48 (2004), pp. 1175-1187. 
[4] N. Q. Balaban, J. Merrin, R. Chait, L. Kowalik, and S. Leibler, Bacterial persistence as a phenotypic switch, Science, 305 (2004), pp. 1622-1625.

[5] M. L. Barclay and E. J. BeGG, Aminoglycoside adaptive resistance: Importance for effective dosage regimens, Drugs, 61 (2001), pp. 713-721.

[6] M. L. Barclay, E. J. Begg, and S. T. Chambers, Adaptive resistance following single doses of gentamicin in a dynamic in vitro model, Antimicrob. Agents Chemother., 36 (1992), pp. 1951-1957.

[7] M. L. Barclay, E. J. Begg, S. T. Chambers, and B. A. Peddie, The effect of aminoglycosideinduced adaptive resistance on the antibacterial activity of other antibiotics against Pseudomonas aeruginosa in vitro, J. Antimicrob. Chemother., 38 (1996), pp. 853-858.

[8] A. Brooun, S. Liu, And K. Lewis, A dose-response study of antibiotic resistance in Pseudomonas aeruginosa biofilms, Antimicrob. Agents Chemother., 44 (2000), pp. 640-646.

[9] W. G. Characklis, Microbial fouling, in Biofilms, W. G. Characklis and K. C. Marshall, eds., Wiley, New York, 1990, pp. 523-584.

[10] N. G. Cogan, Incorporating toxin hypothesis into a mathematical model of persister formation and dynamics, J. Theoret. Biol., 248 (2007), pp. 340-349.

[11] N. G. Cogan, Effects of persister formation on bacterial response to dosing, J. Theoret. Biol., 238 (2006), pp. 694-703.

[12] N. G. Cogan, R. Cortez, And L. Faud, Modeling physiological resistance in bacterial biofilms, Bull. Math. Biol., 67 (2005), pp. 831-853.

[13] G. L. Daikos, Adaptive resistance to aminoglycoside antibiotics from first-exposure downregulation, J. Inf. Diseases, 162 (1990), pp. 414-420.

[14] J. G. Elkins, D. J. Hassett, P. S. Stewart, H. P. Schweizer, and T. R. McDermott, Protective role of catalase in Pseudomonas aeruginosa biofilm resistance to hydrogen peroxide, Appl. Environ. Microbiol., 65 (1999), pp. 4594-4600.

[15] B. Giwercman, E. T. H. Jensen, N. Høiby, A. Kharazmi, and J. W. Costeron, Induction of $\beta$-lactamase production in Pseudomonas aeruginosa biofilm, Antimicrob. Agents Chemother., 35 (1991), pp. 1008-1010.

[16] D. M. Grant and T. R. Brott, Biofilm dosing strategies for biofilm control, in Heat Exchanger Fouling and Cleaning: Fundamentals and Applications, P. Watkinson, H. MüllerSteinhagen, and M. R. Malayeri, eds., ECI Symposium Series RP1, 2003, pp. 310-316.

[17] K. J. Grobe, J. Zahller, and P. S. Stewart, Role of dose concentration in biocide efficacy against Pseudomonas aeruginosa biofilms, J. Ind. Microbiol. Biotechnol., 29 (2002), pp. $10-15$.

[18] B. D. Hoyle and W. J. Costerton, Bacterial resistance to antibiotics: The role of biofilms, Prog. Drug Res., 37 (1991), pp. 91-105.

[19] J. A. Karlowsky, S. A. Zelenitsky, and G. G. Zhanel, Aminoglycoside adaptive resistance, Pharmacotheraphy, 17 (1997), pp. 549-555.

[20] I. Keren, N. Kaldalu, A. Spoering, Y. Wang, and K. Lewis, Persister cells and tolerance to antimicrobials, FEMS Microbiol. Lett., 230 (2004), pp. 13-18.

[21] I. Klapper, P. Gilbert, B. P. Ayati, J. Dockery, and P. S. Stewart, Senescence can explain microbial persistence, Microbiology, 153 (2007), pp. 3623-3630.

[22] K. LEWIS, Riddle of biofilm resistance, Antimicrob. Agents Chemother., 45 (2001), pp. 9991007.

[23] T.-F. C. Mah and G. A. O'Toole, Mechanisms of biofilm resistance to antimicrobial agents, Trends Microbiol., 9 (2001), pp. 34-39.

[24] T.-F. C. Mah, B. Pitts, B. Pellock, G. C. Walker, P. S. Stewart, and G. A. O'Toole, A genetic basis for Pseudomonas aeruginosa biofilm antibiotic resistance, Nature, 426 (2003), pp. 306-310.

[25] S. H. Powell, W. L. Thompson, M. A. Luthe, R. C. Stern, D. A. Grossniklaus, D. D. Bloxham, D. L. Groden, M. R. Jacobs, A. O. DiScennna, H. A. Cash, and J. D. KLINGER, Once-daily vs. continuous aminoglycoside dosing: Efficacy and toxicity in animal and clinical studies of gentamicin, netilmicin, and tobramycin, J. Inf. Diseases, 147 (1983), pp. 918-931.

[26] B. Prakash, B. M. Veeregowda, and G. Krishnappa, Biofilms: A survival strategy of bacteria, Current Sci., 85 (2003), pp. 1299-1307.

[27] J. Pratten, A. W. Smith, and M. WiLson, Response of single species biofilms and microcosm dental plaques to pulsing with chlorhexidine, J. Antimicrob. Chemother., 42 (1998), pp. 453-459.

[28] Research on Microbial Biofilms, Program Announcement PA-03-047, NIH, National Heart, Lung, and Blood Institute, 2002.

[29] F. C. Saller, B. M. Meberg, and K. D. Young, $\beta$-lactam induction of colanic acid gene expression in Escherichia coli, FEMS Microbiol. Lett., 226 (2003), pp. 245-249.

Copyright (c) by SIAM. Unauthorized reproduction of this article is prohibited. 
[30] S. S. SANDERson And P. S. Stewart, Evidence of bacterial adaptation to monochloramine in Pseudomonas aeruginosa biofilms and evaluation of biocide action model, Biotech. Bioengrg., 56 (1997), pp. 201-209.

[31] R. Singh, P. Ray, A. DAS, And M. Sharma, Role of persisters and small-colony variants in antibiotic resistance of planktonic and biofilm-associated Staphylococcus aureus: An in vitro study, J. Med. Microbiol., 58 (2009), pp. 1067-1073.

[32] O. Steuernagel and D. Polani, Optimal Strategies for Fighting Persister Bugs, preprint, 2008; available online from http://arxiv.org/abs/q-bio/0512003.

[33] P. S. Stewart, Private communication, 2006.

[34] P. S. Stewart, Mechanism of antibiotic resistance in bacterial biofilms, Int. J. Med. Microbiol., 292 (2002), pp. 107-113.

[35] P. S. Stewart, A model of biofilm detachment, Biotech. Bioengrg., 41 (1993), pp. 111-117.

[36] P. S. Stewart, M. A. Hamilton, B. R. Goldstein, and B. T. Schneider, Modeling biocide action against biofilms, Biotech. Bioengrg., 49 (1996), pp. 445-455.

[37] B. Szomolay, Analysis of a moving boundary value problem arising in biofilm modelling, Math. Methods Appl. Sci., 31 (2008), pp. 1835-1859.

[38] B. Szomolay, Analysis and Control of a Biofilm Disinfection Model, Ph.D. thesis, Montana State University, Bozeman, MT, 2006.

[39] B. Szomolay, I. Klapper, J. Dockery, and P. S. Stewart, Adaptive responses to antimicrobial agents in biofilms, Environ. Microbiol., 7 (2005), pp. 1186-1191.

[40] G. Tсhobanoglous, F. L. Burton, and H. D. Stensel, Wastewater Engineering: Treatment and Reuse, McGraw-Hill, New York, 2002.

[41] M. Whiteley, M. G. Bangera, R. E. Bumgarner, M. R. Parsek, G. M. Teitzel, S. Lory, And E. P. Greenberg, Gene expression in Pseudomonas aeruginosa biofilms, Nature, 413 (2001), pp. 860-864.

Copyright (c) by SIAM. Unauthorized reproduction of this article is prohibited. 